\title{
PPF Dependent fixed points of generalized Suzuki type contractions via simulation functions
}

\author{
Venkata Ravindranadh Babu GUTTI ${ }^{\mathrm{a}}$, Vinod Kumar MADUGULA ${ }^{\mathrm{b}}$ \\ ${ }^{a}$ Department of Mathematics, Andhra University, Visakhapatnam - 530 003, India. \\ ${ }^{b}$ Department of Mathematics, Andhra University, Visakhapatnam - 530 003, India. \\ Permanent Address : Department of Mathematics, Anil Neerukonda Institute of Technology and Science, Sangivalasa, \\ Visakhapatnam-531 162, India.
}

\begin{abstract}
In this paper, we introduce generalized Suzuki type $Z_{G, \alpha, \mu, \eta}-$ contraction with respect to $\zeta$ by using the notion of $C_{G}$-simulation function introduced by Liu, Ansari, Chandok and Radenović[19] and prove the existence of PPF dependent fixed points in Banach spaces. We draw some corollaries and an example is provided to illustrate our main result.
\end{abstract}

Keywords: $\alpha$-admissible $\mu$-subadmissible $C$-class function Suzuki type contraction Razumikhin class PPF dependent fixed point simulation function $C_{G}$-simulation function.

2010 MSC: 47H10, 54H25.

\section{Introduction and Preliminaries}

Metric fixed point theory is a suggestive area which includes useful methods, directions, and notions for dealing with various problems. In this area, Banach contraction principle is considered as a fundemental result. In this principle, Banach proved the existence of fixed points in complete metric spaces in a particular manner. Due to its importance and way of construction of the proof, many authors attracted and proved its generalizations and extensions by introducing a new function like $\alpha$-admissible mapping, $C$-class function, etc., for more details we refer [1, 2, 3, 12, 13, 16, 22, 24, 26].

\footnotetext{
Email addresses: gvr_babu@hotmail.com (Venkata Ravindranadh Babu GUTTI), dravinodvivek@gmail.com (Vinod Kumar MADUGULA)
} 
Recently, Khojasteh, Shukla and Radenović[1] introduced the notion of simulation function in order to express different contractivity conditions in a simple, unified manner and they obtained some fixed point results. Later, many authors extended and generalized the simulation function by using different types of functions, for more details we refer [15, 17, 19, 21, 23, 25].

Throughout this paper, we denote the real line by $\mathbb{R}, \mathbb{R}^{+}=[0, \infty)$, and $\mathbb{N}$ is the set of all natural numbers, $\mathbb{Z}$ is the set of intergers.

In 2014, Ansari [1] introduced the concept of $C$ - class function and many authors extended and generalized various fixed point results by using $C$-class functions as a main source in complete metric spaces.

Definition 1.1. [1] A mapping $G: \mathbb{R}^{+} \times \mathbb{R}^{+} \rightarrow \mathbb{R}$ is called a $C$-class function if it is continuous and for any $s, t \in \mathbb{R}^{+}$, the function $G$ satisfies the following conditions:

(i) $G(s, t) \leq s$ and

(ii) $G(s, t)=s$ implies that either $s=0$ or $t=0$.

We denote the family of all $C$-class functions by $\Delta$.

Example 1.2. [1] The following functions belong to $\Delta$.

(i) $G(s, t)=s-t$ for all $s, t \in \mathbb{R}^{+}$.

(ii) $G(s, t)=k s$ for all $s, t \in \mathbb{R}^{+}$where $0<k<1$.

(iii) $G(s, t)=\frac{s}{(1+t)^{r}}$ for all $s, t \in \mathbb{R}^{+}$where $r \in \mathbb{R}^{+}$.

(iv) $G(s, t)=s \beta(s)$ for all $s, t \in \mathbb{R}^{+}$where $\beta: \mathbb{R}^{+} \rightarrow[0,1)$ is continuous.

(v) $G(s, t)=s-\phi(s)$ for all $s, t \in \mathbb{R}^{+}$where $\phi: \mathbb{R}^{+} \rightarrow \mathbb{R}^{+}$is continuous and $\phi(t)=0$ if and only if $t=0$.

(vi) $G(s, t)=\operatorname{sh}(s, t)$ for all $s, t \in \mathbb{R}^{+}$where $h: \mathbb{R}^{+} \times \mathbb{R}^{+} \rightarrow \mathbb{R}^{+}$is continuous such that $h(s, t)<1$ for all $s, t \in \mathbb{R}^{+}$.

In 2015, Khojasteh, Shukla and Radenović[1] introduced the simulation function as follows.

Definition 1.3. [11] A function $\zeta: \mathbb{R}^{+} \times \mathbb{R}^{+} \rightarrow \mathbb{R}$ is said to be a simulation function if it satisfies the following conditions:

$\left(\zeta_{1}\right) \zeta(0,0)=0$

$\left(\zeta_{2}\right) \zeta(t, s)<s-t$ for all $t, s>0$

$\left(\zeta_{3}\right)$ if $\left\{t_{n}\right\},\left\{s_{n}\right\}$ are sequences in $(0, \infty)$ such that $\lim _{n \rightarrow \infty} t_{n}=\lim _{n \rightarrow \infty} s_{n}>0$, then $\limsup _{n \rightarrow \infty} \zeta\left(t_{n}, s_{n}\right)<0$.

We denote the set of all simulation functions in the sense of of Definition 1.3 by $Z_{H}$.

Example 1.4. [11, 15] Let $\phi_{i}: \mathbb{R}^{+} \rightarrow \mathbb{R}^{+}$be a continuous function with $\phi_{i}(t)=0$ if and only if $t=0$ for $i=1,2,3$. Then the following functions $\zeta: \mathbb{R}^{+} \times \mathbb{R}^{+} \rightarrow \mathbb{R}$ belong to $Z_{H}$.

(i) $\zeta(t, s)=\frac{s}{s+1}-t$ for all $t, s \in \mathbb{R}^{+}$.

(ii) $\zeta(t, s)=\lambda s-t$ for all $t, s \in \mathbb{R}^{+}$and $0<\lambda<1$.

(iii) $\zeta(t, s)=\phi_{1}(s)-\phi_{2}(t)$ for all $t, s \in \mathbb{R}^{+}$, where $\phi_{1}(t)<t \leq \phi_{2}(t)$ for all $t>0$.

(iv) $\zeta(t, s)=s-\phi_{3}(s)-t$ for all $t, s, \in \mathbb{R}^{+}$.

Definition 1.5. [11] Let $(X, d)$ be a metric space, $T: X \rightarrow X$ be a mapping and $\zeta \in Z_{H}$. Then $T$ is called a $Z_{H}$-contraction with respect to $\zeta$ if

$$
\zeta(d(T x, T y), d(x, y)) \geq 0
$$

for any $x, y \in X$.

Theorem 1.6. [11] Let $(X, d)$ be a complete metric space and $T: X \rightarrow X$ be a $Z_{H}$-contraction with respect to $\zeta$. Then $T$ has a unique fixed point $u$ in $X$ and for every $x_{0} \in X$ the Picard sequence $\left\{x_{n}\right\}$ where $x_{n}=T x_{n-1}$ for any $n \in \mathbb{N}$ converges to the fixed point of $T$. 
Definition 1.7. [16] Let $T$ be a self mapping on $X$ and let $\alpha: X \times X \rightarrow \mathbb{R}^{+}$be a function. We say that $T$ is an $\alpha$-admissible mapping if for any $x, y \in X$ with $\alpha(x, y) \geq 1$ implies $\alpha(T x, T y) \geq 1$.

In 2016, Karapinar 15] introduced the notion of $\alpha$-admissible $Z_{H}$-contraction with respect to the simulation function $\zeta$ and proved the existence of its fixed points in complete metric spaces.

Definition 1.8. [15] Let $T$ be a self-mapping defined on a metric space $(X, d)$. If there exist $\zeta \in Z_{H}$ and $\alpha: X \times X \rightarrow \mathbb{R}^{+}$such that

$$
\zeta(\alpha(x, y) d(T x, T y), d(x, y)) \geq 0
$$

for all $x, y \in X$, then we say that $T$ is an $\alpha$-admissible $Z_{H}$-contraction with respect to $\zeta$.

Definition 1.9. [22] Let $T: X \rightarrow X$ be a mapping and $\alpha: X \times X \rightarrow \mathbb{R}^{+}$be a function. We say that $T$ is an $\alpha$-orbital admissible if

$$
x \in X, \alpha(x, T x) \geq 1 \Longrightarrow \alpha\left(T x, T^{2} x\right) \geq 1 .
$$

Furthermore, $T$ is called a triangular $\alpha$-orbital admissible if $T$ is $\alpha$-orbital admissible and

$$
x, y \in X, \alpha(x, y) \geq 1 \quad \text { and } \quad \alpha(y, T y) \geq 1 \Longrightarrow \alpha(x, T y) \geq 1 .
$$

Theorem 1.10. [15] Let $(X, d)$ be a complete metric space, $\zeta \in Z_{H}$ and let $T: X \rightarrow X$ be an $\alpha-a d m i s s i b l e$ $Z_{H}-$ contraction with respect to $\zeta$. Suppose that

(i) $T$ is triangular $\alpha$-orbital admissible,

(ii) there exists $x_{0} \in X$ such that $\alpha\left(x_{0}, T x_{0}\right) \geq 1$,

(iii) $T$ is continuous.

Then there exists $u \in X$ such that $T u=u$.

In 2017, Kumum, Gopal and Budhia[17] introduced the notion of Suzuki type $Z_{H}$-contraction by combining the Suzuki type contraction and $Z_{H}$ - contraction and proved the existence of its fixed points in complete metric spaces.

Definition 1.11. [17] Let $(X, d)$ be a metric space, $T: X \rightarrow X$ be a mapping and $\zeta \in Z_{H}$. Then $T$ is called a Suzuki type $Z_{H}$-contraction with respect to $\zeta$ if

$$
\frac{1}{2} d(x, T x)<d(x, y) \Longrightarrow \zeta(d(T x, T y), d(x, y)) \geq 0
$$

for any $x, y \in X$ with $x \neq y$.

Definition 1.12. [17] Let $T: X \rightarrow X$ be a mapping and $x_{0} \in X$ be aribitrary. Then $T$ is said to possess property $(K)$ if for a bounded Picard sequence

$x_{n}=T x_{n-1}, n=1,2,3, \ldots$, there exist subsequences $\left\{x_{m_{k}}\right\}$ and $\left\{x_{n_{k}}\right\}$ such that $\lim _{k \rightarrow \infty} d\left(x_{m_{k}}, x_{n_{k}}\right)=C>0$ where $m_{k}>n_{k}>k, k \in \mathbb{N}$ then

$$
\frac{1}{2} d\left(x_{m_{k}-1}, x_{m_{k}}\right)<d\left(x_{m_{k}-1}, x_{n_{k}-1}\right)
$$

holds.

Theorem 1.13. [17] Let $(X, d)$ be a complete metric space, $\zeta \in Z_{H}$ and $T: X \rightarrow X$ be a Suzuki type $Z_{H}$-contraction with respect to $\zeta$. Then $T$ has a unique fixed point $u$ in $X$ and for every $x_{0} \in X$ the Picard sequence $\left\{x_{n}\right\}$ where $x_{n}=T x_{n-1}$ for $n=1,2,3, \ldots$, converges to the fixed point of $T$, provided $T$ has property $(K)$.

In 2018, Padcharoen, Kumum, Saipara and Cahipunya[21] introduced the notion of generalized Suzuki type $Z_{H}-$ contraction and proved the existence of its fixed points in complete metric spaces. 
Definition 1.14. [21] Let $(X, d)$ be a metric space, $T: X \rightarrow X$ be a mapping and $\zeta \in Z_{H}$. Then $T$ is called generalized Suzuki type $Z_{H}$-contraction with respect to $\zeta$ if

$$
\frac{1}{2} d(x, T x)<d(x, y) \Longrightarrow \zeta(d(T x, T y), M(x, y)) \geq 0
$$

for any $x, y \in X$ with $x \neq y$, where

$$
M(x, y)=\max \left\{d(x, y), d(x, T x), d(y, T y), \frac{d(x, T y)+d(y, T x)}{2}\right\} .
$$

Theorem 1.15. [21] Let $(X, d)$ be a complete metric space, $\zeta \in Z_{H}$ and $T$ is a generalized Suzuki type $Z_{H}$-contraction with respect to $\zeta$. Then $T$ has a fixed point.

In 2015, Roldán, Karapınar, Roldán, Martinez[25] modified the Definition 1.3 of simulation function as follows.

Definition 1.16. [25] A function $\zeta: \mathbb{R}^{+} \times \mathbb{R}^{+} \rightarrow \mathbb{R}$ is said to be a simulation function if it satisfies the following conditions:

$\left(\zeta_{4}\right) \zeta(0,0)=0$

$\left(\zeta_{5}\right) \zeta(t, s)<s-t$ for all $t, s>0$;

$\left(\zeta_{6}\right)$ if $\left\{t_{n}\right\},\left\{s_{n}\right\}$ are sequences in $(0, \infty)$ such that $\lim _{n \rightarrow \infty} t_{n}=\lim _{n \rightarrow \infty} s_{n}>0$ and $t_{n}<s_{n}$ then $\limsup _{n \rightarrow \infty} \zeta\left(t_{n}, s_{n}\right)<0$.

Clearly every simulation function in the sense of Definition 1.3 is also a simulation function in the sense of Definition 1.16. Roldán, Karapınar, Roldán, Martinez[25] shown that its converse is not true(Example $3.3,[25])$.

In 2018, Liu, Ansari, Chandok and Radenović[19] generalized the simulation function introduced by Khojasteh, Shukla and Radenović[11] by using $C$-class function as follows.

Definition 1.17. [19] A mapping $G: \mathbb{R}^{+} \times \mathbb{R}^{+} \rightarrow \mathbb{R}$ has the property $C_{G}$ if there exists an $C_{G} \geq 0$ such that (i) $G(s, t)>C_{G}$ implies $s>t$, and

(ii) $G(t, t) \leq C_{G}$ for all $s, t \in \mathbb{R}^{+}$.

Example 1.18. [19] The following functions $G: \mathbb{R}^{+} \times \mathbb{R}^{+} \rightarrow \mathbb{R}$ are functions of $\Delta$ that are from Definition 1.1 and having the property $C_{G}$. For all $s, t \in \mathbb{R}^{+}$,

(i) $G(s, t)=s-t, C_{G}=r, r \in \mathbb{R}^{+}$,

(ii) $G(s, t)=s-\frac{(2+t) t}{1+t}, C_{G}=0$,

(iii) $G(s, t)=\frac{s}{1+k t}, k \geq 1, C_{G}=\frac{r}{1+k}, r \geq 2$.

Definition 1.19. [19]A function $\zeta: \mathbb{R}^{+} \times \mathbb{R}^{+} \rightarrow \mathbb{R}$ is said to be a $C_{G^{-}}$simulation function if it satisfies the following conditions:

$\left(\zeta_{7}\right) \zeta(0,0)=0$

$\left(\zeta_{8}\right) \zeta(t, s)<G(s, t)$ for all $t, s>0$; here function $G: \mathbb{R}^{+} \times \mathbb{R}^{+} \rightarrow \mathbb{R}^{+}$is an element of $\Delta$ which has property $C_{G}$;

$\left(\zeta_{9}\right)$ if $\left\{t_{n}\right\},\left\{s_{n}\right\}$ are sequences in $(0, \infty)$ such that $\lim _{n \rightarrow \infty} t_{n}=\lim _{n \rightarrow \infty} s_{n}>0$ and $t_{n}<s_{n}$ then $\limsup _{n \rightarrow \infty} \zeta\left(t_{n}, s_{n}\right)<C_{G}$.

We denote the set of all $C_{G}$-simulation functions by $Z_{G}$.

Example 1.20. We define $\zeta: \mathbb{R}^{+} \times \mathbb{R}^{+} \rightarrow \mathbb{R}$ by $\zeta(t, s)=\lambda s-t$, where $\lambda \in(0,1)$ and $G: \mathbb{R}^{+} \times \mathbb{R}^{+} \rightarrow \mathbb{R}$ by $G(s, t)=s-t$ for any $s, t \in \mathbb{R}^{+}$.

Clearly $\zeta(0,0)=0$ and $G \in \Delta$ with $C_{G}=0$.

Clearly $\zeta(t, s)=\lambda s-t<s-t=G(s, t)$ and hence $\zeta$ satisfies $\left(\zeta_{8}\right)$.

If $\left\{t_{n}\right\},\left\{s_{n}\right\}$ are sequences in $(0, \infty)$ such that $\lim _{n \rightarrow \infty} t_{n}=\lim _{n \rightarrow \infty} s_{n}=k>0$ 
and $t_{n}<s_{n}$ for all $n \in \mathbb{N}$, then

$\limsup _{n \rightarrow \infty} \zeta\left(t_{n}, s_{n}\right)=\limsup _{n \rightarrow \infty}\left(\lambda s_{n}-t_{n}\right)=\lambda k-k=(\lambda-1) k<0$.

Therefore $\zeta$ satisfies $\left(\zeta_{9}\right)$ and hence $\zeta \in Z_{G}$.

Karapinar, Kumam and Salimi [16] introduced the notion of triangular $\alpha$-admissible mappings as follows.

Definition 1.21. [16] Let $T$ be a self mapping on $X$ and let $\alpha: X \times X \rightarrow \mathbb{R}^{+}$be a function. Then $T$ is said to be a triangular $\alpha$-admissible mapping if for any $x, y, z \in X$,

$$
\begin{aligned}
& \alpha(x, y) \geq 1 \Longrightarrow \alpha(T x, T y) \geq 1 \text { and } \\
& \alpha(x, z) \geq 1, \alpha(z, y) \geq 1 \Longrightarrow \alpha(x, y) \geq 1 .
\end{aligned}
$$

In 1977, Bernfeld, Lakshmikantham and Reddy[7] introduced the concept of fixed point for mappings that have different domains and ranges which is called PPF (Past, Present and Future) dependent fixed point. Furthermore, they gave notion of Banach type contraction for non-self mapping and proved the existence of PPF dependent fixed points in the Razumikhin class for Banach type contraction mappings, for further details we refer [5, 6, 9, 10, 14, 18].

Let $\left(E,\|\cdot\|_{E}\right)$ be a Banach space and we denote it simply by $E$. Let $I=[a, b] \subseteq \mathbb{R}$ and $E_{0}=C(I, E)$, the set of all continuous functions on $I$ equipped with the supremum norm $\|\cdot\|_{E_{0}}$ and we define it by $\|\phi\|_{E_{0}}=\sup _{a \leq t \leq b}\|\phi(t)\|_{E}$ for $\phi \in E_{0}$.

For a fixed $c \in I$, the Razumikhin class $R_{c}$ of functions in $E_{0}$ is defined by $R_{c}=\left\{\phi \in E_{0} /\|\phi\|_{E_{0}}=\|\phi(c)\|_{E}\right\}$. Clearly every constant function from $I$ to $E$ belongs to $R_{c}$ so that $R_{c}$ is a non-empty subset of $E_{0}$.

Definition 1.22. [7] Let $R_{c}$ be the Razumikhin class of continuous functions in $E_{0}$. We say that

(i) the class $R_{c}$ is algebraically closed with respect to the difference if $\phi-\psi \in R_{c}$ whenever $\phi, \psi \in R_{c}$.

(ii) the class $R_{c}$ is topologically closed if it is closed with respect to the topology on $E_{0}$ by the norm $\|\cdot\|_{E_{0}}$.

The Razumikhin class of functions $R_{c}$ has the following properties.

Theorem 1.23. [4] Let $R_{c}$ be the Razumikhin class of functions in $E_{0}$. Then

(i) for any $\phi \in R_{c}$ and $\alpha \in \mathbb{R}$, we have $\alpha \phi \in R_{c}$.

(ii) the Razumikhin class $R_{c}$ is topologically closed with respect to the norm defined on $E_{0}$.

(iii) $\underset{c \in[a, b]}{\cap R_{c}}=\left\{\phi \in E_{0} / \phi: I \rightarrow E\right.$ is constant $\}$.

Definition 1.24. [7] Let $T: E_{0} \rightarrow E$ be a mapping. A function $\phi \in E_{0}$ is said to be a PPF dependent fixed point of $T$ if $T \phi=\phi(c)$ for some $c \in I$.

Definition 1.25. [7] Let $T: E_{0} \rightarrow E$ be a mapping. Then $T$ is called a Banach type contraction if there exists $k \in[0,1)$ such that

$\|T \phi-T \psi\|_{E} \leq k\|\phi-\psi\|_{E_{0}}$ for all $\phi, \psi \in E_{0}$.

Theorem 1.26. [7] Let $T: E_{0} \rightarrow E$ be a Banach type contraction. Let $R_{c}$ be algebraically closed with respect to the difference and topologically closed. Then $T$ has a unique PPF dependent fixed point in $R_{c}$.

Definition 1.27. Let $c \in I$. Let $T: E_{0} \rightarrow E$ and $\alpha: E \times E \rightarrow \mathbb{R}^{+}$be two functions. Then $T$ is said to be a $\alpha_{c}-$ admissible mapping if for any $\phi, \psi \in E_{0}$,

$$
\alpha(\phi(c), \psi(c)) \geq 1 \Longrightarrow \alpha(T \phi, T \psi) \geq 1 .
$$

Definition 1.28. Let $c \in I$. Let $T: E_{0} \rightarrow E$ and $\mu: E \times E \rightarrow \mathbb{R}^{+}$be two functions. Then $T$ is said to be a $\mu_{c}-$ subadmissible mapping if for any $\phi, \psi \in E_{0}$,

$$
\mu(\phi(c), \psi(c)) \leq 1 \Longrightarrow \mu(T \phi, T \psi) \leq 1 .
$$


Ćirić, Alsulami, Salimi and Vetro[ 8 introduced the concept of triangular $\alpha_{c}$-admissible mapping with respect to $\mu_{c}$ as follows.

Definition 1.29. [8] Let $c \in I$ and $T: E_{0} \rightarrow E$. Let $\alpha, \mu: E \times E \rightarrow \mathbb{R}^{+}$be two functions. Then $T$ is said to be a triangular $\alpha_{c}$-admissible mapping with respect to $\mu_{c}$ if for any $\phi, \psi, \varphi \in E_{0}$,

$$
\left\{\begin{array}{c}
(i) \alpha(\phi(c), \psi(c)) \geq \mu(\phi(c), \psi(c)) \Longrightarrow \alpha(T \phi, T \psi) \geq \mu(T \phi, T \psi) \\
\text { and } \\
(i i) \alpha(\phi(c), \psi(c)) \geq \mu(\phi(c), \psi(c)), \alpha(\psi(c), \varphi(c)) \geq \mu(\psi(c), \varphi(c)) \\
\Longrightarrow \alpha(\phi(c), \varphi(c)) \geq \mu(\phi(c), \varphi(c)) .
\end{array}\right.
$$

Note that if $\mu(x, y)=1$ for any $x, y \in E$, then we say that $T$ is a triangular $\alpha_{c}-$ admissible mapping and if $\alpha(x, y)=1$ for any $x, y \in E$, then we say that $T$ is a triangular $\mu_{c}-$ subadmissible mapping.

Lemma 1.30. [8] Let $T$ be a triangular $\alpha_{c}$-admissible mapping with respect to $\mu_{c}$. We define the sequence $\left\{\phi_{n}\right\}$ by $T \phi_{n}=\phi_{n+1}(c)$ for all $n \in \mathbb{N} \cup\{0\}$, where $\phi_{0} \in R_{c}$ is such that $\alpha\left(\phi_{0}(c), T \phi_{0}\right) \geq \mu\left(\phi_{0}(c), T \phi_{0}\right)$. Then $\alpha\left(\phi_{m}(c), \phi_{n}(c)\right) \geq \mu\left(\phi_{m}(c), \phi_{n}(c)\right)$ for all $m, n \in \mathbb{N}$ with $m<n$.

If $\mu(x, y)=1$ for any $x, y \in E$ in Lemma 1.30 , we get the following lemma.

Lemma 1.31. Let $T$ be a triangular $\alpha_{c}$-admissible mapping. We define the sequence $\left\{\phi_{n}\right\}$ by $T \phi_{n}=\phi_{n+1}(c)$ for all $n \in \mathbb{N} \cup\{0\}$, where $\phi_{0} \in R_{c}$ is such that $\alpha\left(\phi_{0}(c), T \phi_{0}\right) \geq 1$. Then $\alpha\left(\phi_{m}(c), \phi_{n}(c)\right) \geq 1$ for all $m, n \in \mathbb{N}$ with $m<n$.

If $\alpha(x, y)=1$ for any $x, y \in E$ in Lemma 1.30 , we get the following lemma.

Lemma 1.32. Let $T$ be a triangular $\mu_{c}$-subadmissible mapping. We define the sequence $\left\{\phi_{n}\right\}$ by $T \phi_{n}=$ $\phi_{n+1}(c)$ for all $n \in \mathbb{N} \cup\{0\}$, where $\phi_{0} \in R_{c}$ is such that $\mu\left(\phi_{0}(c), T \phi_{0}\right) \leq 1$. Then $\mu\left(\phi_{m}(c), \phi_{n}(c)\right) \leq 1$ for all $m, n \in \mathbb{N}$ with $m<n$.

We use the following proposition to prove Lemma 1.34 .

Proposition 1.33. If $\left\{a_{n}\right\}$ and $\left\{b_{n}\right\}$ are two real sequences, $\left\{b_{n}\right\}$ is bounded, then $\lim \inf \left(a_{n}+b_{n}\right) \leq$ $\lim \inf a_{n}+\lim \sup b_{n}$.

Lemma 1.34. Let $\left\{\phi_{n}\right\}$ be a sequence in $E_{0}$ such that $\left\|\phi_{n}-\phi_{n+1}\right\|_{E_{0}} \rightarrow 0$ as $n \rightarrow \infty$. If $\left\{\phi_{n}\right\}$ is not a Cauchy sequence, then there exists an $\epsilon>0$ and two subsequences $\left\{\phi_{m_{k}}\right\}$ and $\left\{\phi_{n_{k}}\right\}$ of $\left\{\phi_{n}\right\}$ with $m_{k}>n_{k}>k$ such that

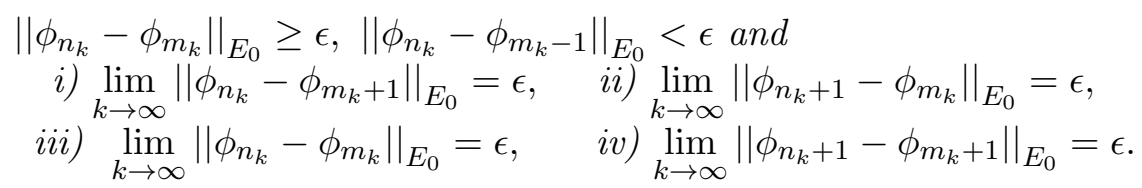

Proof. If $\left\{\phi_{n}\right\}$ is not a Cauchy sequence then there exists an $\epsilon>0$ and two subsequences $\left\{\phi_{m_{k}}\right\}$ and $\left\{\phi_{n_{k}}\right\}$ with $m_{k}>n_{k}>k$ satisfying

$$
\left\|\phi_{n_{k}}-\phi_{m_{k}}\right\|_{E_{0}} \geq \epsilon
$$

We choose $m_{k}$, the least positive integer satisfying (11). Then we have

$$
\left\|\phi_{n_{k}}-\phi_{m_{k}}\right\|_{E_{0}} \geq \epsilon \text { and }\left\|\phi_{n_{k}}-\phi_{m_{k}-1}\right\|_{E_{0}}<\epsilon .
$$

We now prove (i).

By triangular inequality we have

$\epsilon \leq\left\|\phi_{n_{k}}-\phi_{m_{k}}\right\|_{E_{0}} \leq\left\|\phi_{n_{k}}-\phi_{m_{k}+1}\right\|_{E_{0}}+\left\|\phi_{m_{k}+1}-\phi_{m_{k}}\right\|_{E_{0}}$. 
Now by applying Proposition 1.33 with $a_{k}=\left\|\phi_{n_{k}}-\phi_{m_{k}+1}\right\|_{E_{0}}$ and

$b_{k}=\left\|\phi_{m_{k}+1}-\phi_{m_{k}}\right\|_{E_{0}}$ we have

$$
\epsilon \leq \liminf _{k \rightarrow \infty}\left\|\phi_{n_{k}}-\phi_{m_{k}+1}\right\|_{E_{0}} .
$$

By triangular inequality we have

$$
\text { (since }\left\|\phi_{n}-\phi_{n+1}\right\|_{E_{0}} \rightarrow 0 \text { as } n \rightarrow \infty \text { ) }
$$

$\left\|\phi_{n_{k}}-\phi_{m_{k}+1}\right\|_{E_{0}} \leq\left\|\phi_{n_{k}}-\phi_{m_{k}-1}\right\|_{E_{0}}+\left\|\phi_{m_{k}-1}-\phi_{m_{k}}\right\|_{E_{0}}$

$$
<\epsilon+\left\|\phi_{m_{k}-1}-\phi_{m_{k}}\right\|_{E_{0}} \text {. (by 12p) }
$$

On applying limit superior as $k \rightarrow \infty$ we get

$$
\limsup _{k \rightarrow \infty}\left\|\phi_{n_{k}}-\phi_{m_{k}+1}\right\|_{E_{0}} \leq \epsilon .
$$

From 13) and 14 we get

$$
\text { (since }\left\|\phi_{n}-\phi_{n+1}\right\|_{E_{0}} \rightarrow 0 \text { as } n \rightarrow \infty \text { ) }
$$

$\epsilon \leq \liminf _{k \rightarrow \infty}\left\|\phi_{n_{k}}-\phi_{m_{k}+1}\right\|_{E_{0}} \leq \limsup _{k \rightarrow \infty}\left\|\phi_{n_{k}}-\phi_{m_{k}+1}\right\|_{E_{0}} \leq \epsilon$.

Therefore

$$
\lim _{k \rightarrow \infty}\left\|\phi_{n_{k}}-\phi_{m_{k}+1}\right\|_{E_{0}}=\epsilon
$$

Hence (i) holds.

We now prove (ii).

By triangular inequality we have

$\epsilon \leq\left\|\phi_{n_{k}}-\phi_{m_{k}}\right\|_{E_{0}} \leq\left\|\phi_{n_{k}}-\phi_{n_{k}+1}\right\|_{E_{0}}+\left\|\phi_{n_{k}+1}-\phi_{m_{k}}\right\|_{E_{0}}$.

Now by applying Proposition 1.33 with $a_{k}=\left\|\phi_{n_{k}+1}-\phi_{m_{k}}\right\|_{E_{0}}$ and

$b_{k}=\left\|\phi_{n_{k}}-\phi_{n_{k}+1}\right\|_{E_{0}}$ we have

$$
\epsilon \leq \liminf _{k \rightarrow \infty}\left\|\phi_{n_{k}+1}-\phi_{m_{k}}\right\|_{E_{0}} .
$$

By triangular inequality we have

$$
\text { (since } \left.\left\|\phi_{n}-\phi_{n+1}\right\|_{E_{0}} \rightarrow 0 \text { as } n \rightarrow \infty\right)
$$

$\left\|\phi_{n_{k}+1}-\phi_{m_{k}}\right\|_{E_{0}} \leq\left\|\phi_{n_{k}+1}-\phi_{n_{k}}\right\|_{E_{0}}+\left\|\phi_{n_{k}}-\phi_{m_{k}+1}\right\|_{E_{0}}+\left\|\phi_{m_{k}+1}-\phi_{m_{k}}\right\|_{E_{0}}$.

On applying limit superior as $k \rightarrow \infty$ we get

$$
\limsup _{k \rightarrow \infty}\left\|\phi_{n_{k}+1}-\phi_{m_{k}}\right\|_{E_{0}} \leq \epsilon .
$$

From (16) and (17) we get

$$
\text { (from } 15 \text { and }\left\|\phi_{n}-\phi_{n+1}\right\|_{E_{0}} \rightarrow 0 \text { as } n \rightarrow \infty \text { ) }
$$

$\epsilon \leq \liminf _{k \rightarrow \infty}\left\|\phi_{n_{k}+1}-\phi_{m_{k}}\right\|_{E_{0}} \leq \limsup _{k \rightarrow \infty}\left\|\phi_{n_{k}+1}-\phi_{m_{k}}\right\|_{E_{0}} \leq \epsilon$.

Therefore

$$
\lim _{k \rightarrow \infty}\left\|\phi_{n_{k}+1}-\phi_{m_{k}}\right\|_{E_{0}}=\epsilon
$$

This proves (ii).

We now prove (iii).

From (11) we have $\left\|\phi_{n_{k}}-\phi_{m_{k}}\right\|_{E_{0}} \geq \epsilon$.

On applying limit inferior as $k \rightarrow \infty$ we get

$$
\liminf _{k \rightarrow \infty}\left\|\phi_{n_{k}}-\phi_{m_{k}}\right\|_{E_{0}} \geq \epsilon
$$

By triangular inequality we have

$\left\|\phi_{n_{k}}-\phi_{m_{k}}\right\|_{E_{0}} \leq\left\|\phi_{n_{k}}-\phi_{n_{k}+1}\right\|_{E_{0}}+\left\|\phi_{n_{k}+1}-\phi_{m_{k}}\right\|_{E_{0}}$.

On applying limit superior as $k \rightarrow \infty$ we get

$$
\limsup _{k \rightarrow \infty}\left\|\phi_{n_{k}}-\phi_{m_{k}}\right\|_{E_{0}} \leq \epsilon
$$


From 19 and 20 we get

$$
\text { (from } \left.18 \text { and }\left\|\phi_{n}-\phi_{n+1}\right\|_{E_{0}} \rightarrow 0 \text { as } n \rightarrow \infty\right)
$$

$\epsilon \leq \liminf _{k \rightarrow \infty}\left\|\phi_{n_{k}}-\phi_{m_{k}}\right\|_{E_{0}} \leq \limsup _{k \rightarrow \infty}\left\|\phi_{n_{k}}-\phi_{m_{k}}\right\|_{E_{0}} \leq \epsilon$.

Therefore

$$
\lim _{k \rightarrow \infty}\left\|\phi_{n_{k}}-\phi_{m_{k}}\right\|_{E_{0}}=\epsilon
$$

Hence (iii) holds.

We now prove (iv).

By triangular inequality we have

$\epsilon \leq\left\|\phi_{n_{k}}-\phi_{m_{k}}\right\|_{E_{0}} \leq\left\|\phi_{n_{k}}-\phi_{n_{k}+1}\right\|_{E_{0}}+\left\|\phi_{n_{k}+1}-\phi_{m_{k}+1}\right\|_{E_{0}}+\left\|\phi_{m_{k}+1}-\phi_{m_{k}}\right\|_{E_{0}}$.

Now by applying Proposition 1.33 with $a_{k}=\left\|\phi_{n_{k}+1}-\phi_{m_{k}+1}\right\|_{E_{0}}$ and

$b_{k}=\left\|\phi_{n_{k}}-\phi_{n_{k}+1}\right\|_{E_{0}}+\left\|\phi_{m_{k}+1}-\phi_{m_{k}}\right\|_{E_{0}}$ we have

$$
\epsilon \leq \liminf _{k \rightarrow \infty}\left\|\phi_{n_{k}+1}-\phi_{m_{k}+1}\right\|_{E_{0}} .
$$

By triangular inequality we have

$$
\text { (since } \left.\left\|\phi_{n}-\phi_{n+1}\right\|_{E_{0}} \rightarrow 0 \text { as } n \rightarrow \infty\right)
$$

$\left\|\phi_{n_{k}+1}-\phi_{m_{k}+1}\right\|_{E_{0}} \leq\left\|\phi_{n_{k}+1}-\phi_{n_{k}}\right\|_{E_{0}}+\left\|\phi_{n_{k}}-\phi_{m_{k}}\right\|_{E_{0}}+\left\|\phi_{m_{k}}-\phi_{m_{k}+1}\right\|_{E_{0}}$.

On applying limit superior as $k \rightarrow \infty$ we get

$$
\limsup _{k \rightarrow \infty}\left\|\phi_{n_{k}+1}-\phi_{m_{k}+1}\right\|_{E_{0}} \leq \epsilon .
$$

From $(22)$ and $(23)$ we get

$$
\text { (from } 21 \text { and }\left\|\phi_{n}-\phi_{n+1}\right\|_{E_{0}} \rightarrow 0 \text { as } n \rightarrow \infty \text { ) }
$$

$\epsilon \leq \liminf _{k \rightarrow \infty}\left\|\phi_{n_{k}+1}-\phi_{m_{k}+1}\right\|_{E_{0}} \leq \limsup _{k \rightarrow \infty}\left\|\phi_{n_{k}+1}-\phi_{m_{k}+1}\right\|_{E_{0}} \leq \epsilon$

Therefore

$$
\lim _{k \rightarrow \infty}\left\|\phi_{n_{k}+1}-\phi_{m_{k}+1}\right\|_{E_{0}}=\epsilon
$$

so that (iv) holds.

In Section 2, we introduce different types of Suzuki type $Z_{H}$-contractions ( $Z_{G}$-contractions) by using simulation functions in $Z_{H}\left(Z_{G}.\right)$ Also, we define generalized Suzuki type $Z_{G, \alpha, \mu, \eta}$-contraction with respect to $\zeta$ in Banach spaces. In Section 3, we prove the existence of PPF dependent fixed points of generalized Suzuki type $Z_{G, \alpha, \mu, \eta}$-contraction with respect to $\zeta$. In Section 4 we draw some corollaries and an example is provided to illustrate our main result.

\section{Suzuki type $Z_{H}-$ contractions}

We denote

$\Psi=\left\{\eta \mid \eta: \mathbb{R}^{+} \rightarrow \mathbb{R}^{+}\right.$is continuous, nondecreasing and

$$
\eta(t)=0 \Longleftrightarrow t=0\} \text {. }
$$

Definition 2.1. Let $c \in I$. Let $T: E_{0} \rightarrow E$ be a function and $\zeta \in Z_{H}$. Then $T$ is called a $Z_{H}$-contraction with respect to $\zeta$ if

$$
\zeta\left(\|T \phi-T \psi\|_{E},\|\phi-\psi\|_{E_{0}}\right) \geq 0
$$

for any $\phi, \psi \in E_{0}$.

Remark 2.2. It is clear from the definition of simulation function that $\zeta(t, s)<0$ for all $t \geq s>0$. Therefore, if $T$ is a $Z_{H}$-contraction with respect to $\zeta$ then

$$
\|T \phi-T \psi\|_{E}<\|\phi-\psi\|_{E_{0}}
$$

for any $\phi, \psi \in E_{0}$. Therefore every $Z_{H}$-contraction mapping is contractive and hence it is continuous. 
Definition 2.3. Let $c \in I$. Let $T: E_{0} \rightarrow E$ be a function and $\zeta \in Z_{H}$. Then $T$ is called Suzuki type $Z_{H}-$ contraction with respect to $\zeta$ if

$$
\frac{1}{2}\|\phi(c)-T \phi\|_{E}<\|\phi-\psi\|_{E_{0}} \Longrightarrow \zeta\left(\|T \phi-T \psi\|_{E},\|\phi-\psi\|_{E_{0}}\right) \geq 0
$$

for any $\phi, \psi \in E_{0}$ with $\phi \neq \psi$.

Remark 2.4. It is clear from the definition of simulation function that $\zeta(t, s)<0$ for all $t \geq s>0$. Therefore, if $T$ is a Suzuki type $Z_{H}$-contraction with respect to $\zeta$ then

$$
\frac{1}{2}\|\phi(c)-T \phi\|_{E}<\|\phi-\psi\|_{E_{0}} \Longrightarrow\|T \phi-T \psi\|_{E}<\|\phi-\psi\|_{E_{0}}
$$

for any $\phi, \psi \in E_{0}$ with $\phi \neq \psi$.

Definition 2.5. Let $c \in I$. Let $T: E_{0} \rightarrow E$ be a function and $\zeta \in Z_{H}$. Then $T$ is called generalized Suzuki type $Z_{H}$-contraction with respect to $\zeta$ if

$$
\frac{1}{2}\|\phi(c)-T \phi\|_{E}<\|\phi-\psi\|_{E_{0}} \Longrightarrow \zeta\left(\|T \phi-T \psi\|_{E}, M(\phi, \psi)\right) \geq 0
$$

for any $\phi, \psi \in E_{0}$ with $\phi \neq \psi$, where

$M(\phi, \psi)=\max \left\{\|\phi-\psi\|_{E_{0}},\|\phi(c)-T \phi\|_{E},\|\psi(c)-T \psi\|_{E}\right.$,

$$
\left.\frac{\|\phi(c)-T \psi\|_{E}+\|\psi(c)-T \phi\|_{E}}{2}\right\} .
$$

Remark 2.6. It is clear from the definition of simulation function that $\zeta(t, s)<0$ for all $t \geq s>0$. Therefore, if $T$ is a generalized Suzuki type $Z_{H}$-contraction with respect to $\zeta$ then

$$
\frac{1}{2}\|\phi(c)-T \phi\|_{E}<\|\phi-\psi\|_{E_{0}} \Longrightarrow\|T \phi-T \psi\|_{E}<M(\phi, \psi)
$$

for any $\phi, \psi \in E_{0}$ with $\phi \neq \psi$, where

$M(\phi, \psi)=\max \left\{\|\phi-\psi\|_{E_{0}},\|\phi(c)-T \phi\|_{E},\|\psi(c)-T \psi\|_{E}\right.$,

$$
\left.\frac{\|\phi(c)-T \psi\|_{E}+\|\psi(c)-T \phi\|_{E}}{2}\right\} \text {. }
$$

Definition 2.7. Let $c \in I$. Let $T: E_{0} \rightarrow E$ be a function and $\zeta \in Z_{H}$. If there exists $\alpha: E \times E \rightarrow \mathbb{R}^{+}$such that

$$
\begin{aligned}
\frac{1}{2}\|\phi(c)-T \phi\|_{E}< & \|\phi-\psi\|_{E_{0}} \Longrightarrow \\
& \zeta\left(\alpha(\phi(c), \psi(c))\|T \phi-T \psi\|_{E}, M(\phi, \psi)\right) \geq 0
\end{aligned}
$$

for any $\phi, \psi \in E_{0}$ with $\phi \neq \psi$, where

$$
\begin{aligned}
& M(\phi, \psi)=\max \left\{\|\phi-\psi\|_{E_{0}},\|\phi(c)-T \phi\|_{E},\|\psi(c)-T \psi\|_{E},\right. \\
& \left.\frac{\|\phi(c)-T \psi\|_{E}+\|\psi(c)-T \phi\|_{E}}{2}\right\},
\end{aligned}
$$

then we say that $T$ is a generalized Suzuki type $Z_{H, \alpha}$-contraction with respect to $\zeta$.

Definition 2.8. Let $c \in I$. Let $T: E_{0} \rightarrow E$ be a function and $\zeta \in Z_{H}$. If there exist $\alpha: E \times E \rightarrow \mathbb{R}^{+}$and $\eta \in \Psi$ such that

$$
\begin{aligned}
& \frac{1}{2}\|\phi(c)-T \phi\|_{E}<\|\phi-\psi\|_{E_{0}} \Longrightarrow \\
& \quad \zeta\left(\alpha(\phi(c), \psi(c)) \eta\left(\|T \phi-T \psi\|_{E}\right), \eta(M(\phi, \psi))\right) \geq 0
\end{aligned}
$$

for any $\phi, \psi \in E_{0}$ with $\phi \neq \psi$, where

$M(\phi, \psi)=\max \left\{\|\phi-\psi\|_{E_{0}},\|\phi(c)-T \phi\|_{E},\|\psi(c)-T \psi\|_{E}\right.$,

$$
\left.\frac{\|\phi(c)-T \psi\|_{E}+\|\psi(c)-T \phi\|_{E}}{2}\right\},
$$

then we say that $T$ is a generalized Suzuki type $Z_{H, \alpha, \eta}$-contraction with respect to $\zeta$.

Remark 2.9. If $\eta$ is the identity mapping in Definition 2.8 then $T$ is a generalized Suzuki type $Z_{H, \alpha}-$ contraction with respect to $\zeta$. 
Definition 2.10. Let $c \in I$. Let $T: E_{0} \rightarrow E$ be a function and $\zeta \in Z_{G}$. If there exist $\alpha, \mu: E \times E \rightarrow \mathbb{R}^{+}$ and $\eta \in \Psi$ such that

$$
\begin{aligned}
& \frac{1}{2} \mu(\phi(c), \psi(c))\|\phi(c)-T \phi\|_{E}<\|\phi-\psi\|_{E_{0}} \Longrightarrow \\
& \quad \zeta\left(\alpha(\phi(c), \psi(c)) \eta\left(\|T \phi-T \psi\|_{E}\right), \eta(M(\phi, \psi))\right) \geq C_{G}
\end{aligned}
$$

for any $\phi, \psi \in E_{0}$ with $\phi \neq \psi$, where

$M(\phi, \psi)=\max \left\{\|\phi-\psi\|_{E_{0}},\|\phi(c)-T \phi\|_{E},\|\psi(c)-T \psi\|_{E}\right.$,

$$
\left.\frac{\|\phi(c)-T \psi\|_{E}+\|\psi(c)-T \phi\|_{E}}{2}\right\}
$$

then we say that $T$ is a generalized Suzuki type $Z_{G, \alpha, \mu, \eta}$-contraction with respect to $\zeta$.

Remark 2.11. If $T$ is a generalized Suzuki type $Z_{G, \alpha, \mu, \eta}$-contraction with respect to $\zeta$ then

$$
\begin{gathered}
\frac{1}{2} \mu(\phi(c), \psi(c))\|\phi(c)-T \phi\|_{E}<\|\phi-\psi\|_{E_{0}} \Longrightarrow \\
\left.\alpha(\phi(c), \psi(c)) \eta\left(\|T \phi-T \psi\|_{E}\right)<\eta(M(\phi, \psi))\right)
\end{gathered}
$$

for any $\phi, \psi \in E_{0}$ with $\phi \neq \psi$, where

$M(\phi, \psi)=\max \left\{\|\phi-\psi\|_{E_{0}},\|\phi(c)-T \phi\|_{E},\|\psi(c)-T \psi\|_{E}\right.$,

$$
\left.\frac{\|\phi(c)-T \psi\|_{E}+\|\psi(c)-T \phi\|_{E}}{2}\right\} .
$$

For, we assume that $M(\phi, \psi)>0$. Then $\eta(M(\phi, \psi))>0$.

If there exist $\phi, \psi \in E_{0}$ such that either $\alpha(\phi(c), \psi(c))=0$ or $\|T \phi-T \psi\|_{E}=0$ then the inequality (34) is trivial.

Suppose that $\alpha(\phi(c), \psi(c)) \neq 0$ and $\|T \phi-T \psi\|_{E} \neq 0$ for any $\phi, \psi \in E_{0}$. By $\left(\zeta_{8}\right)$, we get

$C_{G} \leq \zeta\left(\alpha(\phi(c), \psi(c)) \eta\left(\|T \phi-T \psi\|_{E}\right), \eta(M(\phi, \psi))\right)$

$<G\left(\eta(M(\phi, \psi)), \alpha(\phi(c), \psi(c)) \eta\left(\|T \phi-T \psi\|_{E}\right)\right)$.

Now from (i) of Definition 1.17 of property $C_{G}$, we get the inequality (34).

Remark 2.12. (i) If $\mu(x, y)=1$ for any $x, y \in E$ in the inequality (33) then $T$ is called a generalized Suzuki type $Z_{G, \alpha, \eta}$-contraction with respect to $\zeta$.

(ii) If $\alpha(x, y)=1$ and $\mu(x, y)=1$ for any $x, y \in E$ in the inequality (33)

then Tis called a generalized Suzuki type $Z_{G, \eta}$-contraction with respect to $\zeta$.

(iii) If $\alpha(x, y)=1=\mu(x, y)$ for any $x, y \in E$ and $\eta=$ identity in the

inequality (33) then $T$ is called a generalized Suzuki type $Z_{G}$-contraction with respect to $\zeta$.

\section{Existence of PPF dependent fixed points}

Theorem 3.1. Let $c \in I$. Let $T: E_{0} \rightarrow E$ be a function satisfying the following conditions:

(i) $T$ is a generalized Suzuki type $Z_{G, \alpha, \mu, \eta}-$ contraction with respect to $\zeta$,

(ii) $T$ is a triangular $\alpha_{c}-$ admissible mapping and triangular $\mu_{c}-$ subadmissible mapping,

(iii) $R_{c}$ is algebraically closed with respect to the difference,

(iv) if $\left\{\phi_{n}\right\}$ is a sequence in $E_{0}$ such that $\phi_{n} \rightarrow \phi$ as $n \rightarrow \infty$, $\alpha\left(\phi_{n}(c), \phi_{n+1}(c)\right) \geq 1$ and $\mu\left(\phi_{n}(c), \phi_{n+1}(c)\right) \leq 1$ for any $n \in \mathbb{N} \cup\{0\}$

then $\alpha\left(\phi_{n}(c), \phi(c)\right) \geq 1$ and $\mu\left(\phi_{n}(c), \phi(c)\right) \leq 1$ for any $n \in \mathbb{N} \cup\{0\}$ and

(v) there exists $\phi_{0} \in R_{c}$ such that $\alpha\left(\phi_{0}(c), T \phi_{0}\right) \geq 1$ and $\mu\left(\phi_{0}(c), T \phi_{0}\right) \leq 1$.

Then $T$ has a PPF dependent fixed point in $R_{c}$.

Moreover, if $\alpha(x, y) \geq 1, \mu(x, y) \leq 1$ for any $x, y \in E$ and if $T$ is one-one then $T$ has a unique PPF dependent fixed point in $R_{c}$.

Proof. From (v), we have $\phi_{0} \in R_{c}$ such that $\alpha\left(\phi_{0}(c), T \phi_{0}\right) \geq 1$ and $\mu\left(\phi_{0}(c), T \phi_{0}\right) \leq 1$. Let $\left\{\phi_{n}\right\}$ be a sequence in $R_{c}$ defined by

$$
T \phi_{n}=\phi_{n+1}(c) \text { and }\left\|\phi_{n+1}-\phi_{n}\right\|_{E_{0}}=\left\|\phi_{n+1}(c)-\phi_{n}(c)\right\|_{E},
$$


for any $n=0,1,2,3 \ldots$

Since $T$ is traingular $\alpha_{c}$-admissible and triangular $\mu_{c}$-subadmissible mappings, by Lemma 1.31 and Lemma 1.32 we have

$$
\alpha\left(\phi_{m}(c), \phi_{n}(c)\right) \geq 1 \quad \text { and } \quad \mu\left(\phi_{m}(c), \phi_{n}(c)\right) \leq 1
$$

for any $m, n \in \mathbb{N}$ with $m<n$.

If there exists $n \in \mathbb{N} \cup\{0\}$ such that $\phi_{n}=\phi_{n+1}$ then $T \phi_{n}=\phi_{n+1}(c)=\phi_{n}(c)$ and hence $\phi_{n} \in R_{c}$ is a PPF dependent fixed point of $T$.

Suppose that $\phi_{n} \neq \phi_{n+1}$ for any $n \in \mathbb{N} \cup\{0\}$.

We consider

$$
\begin{gathered}
M\left(\phi_{n}, \phi_{n+1}\right)=\max \left\{\left\|\phi_{n}-\phi_{n+1}\right\|_{E_{0},\left\|\phi_{n}(c)-T \phi_{n}\right\|_{E},\left\|\phi_{n+1}(c)-T \phi_{n+1}\right\|_{E},}^{\left.\frac{\left\|\phi_{n}(c)-T \phi_{n+1}\right\|_{E}+\left\|\phi_{n+1}(c)-T \phi_{n}\right\|_{E}}{2}\right\}}\right. \\
=\max \left\{\left.\left\|\phi_{n}-\phi_{n+1}\right\|\right|_{E_{0}},\left\|\phi_{n+1}-\phi_{n+2}\right\|_{E_{0}}\right\} .
\end{gathered}
$$

Clearly

$$
\begin{aligned}
\frac{1}{2} \mu\left(\phi_{n}(c), \phi_{n+1}(c)\right)\left\|\phi_{n}(c)-T \phi_{n}\right\|_{E} & \leq \frac{1}{2}\left\|\phi_{n}(c)-\phi_{n+1}(c)\right\|_{E} \\
& =\frac{1}{2}\left\|\phi_{n}-\phi_{n+1}\right\|_{E_{0}} \\
& <\left\|\phi_{n}-\phi_{n+1}\right\|_{E_{0}} .
\end{aligned}
$$

From (33), we have

$$
C_{G} \leq \zeta\left(\alpha\left(\phi_{n}(c), \phi_{n+1}(c)\right) \eta\left(\left\|T \phi_{n}-T \phi_{n+1}\right\|_{E}\right), \eta\left(M\left(\phi_{n}, \phi_{n+1}\right)\right)\right) .
$$

Suppose that $M\left(\phi_{n}, \phi_{n+1}\right)=\left\|\phi_{n+1}-\phi_{n+2}\right\|_{E_{0}}$.

Clearly $\alpha\left(\phi_{n}(c), \phi_{n+1}(c)\right) \eta\left(\left\|T \phi_{n}-T \phi_{n+1}\right\|_{E}\right)>0$ and $\eta\left(M\left(\phi_{n}, \phi_{n+1}\right)\right)>0$.

From (38), we have

$C_{G} \leq \zeta\left(\alpha\left(\phi_{n}(c), \phi_{n+1}(c)\right) \eta\left(\left\|\phi_{n+1}-\phi_{n+2}\right\|_{E_{0}}\right), \eta\left(\left\|\phi_{n+1}-\phi_{n+2}\right\|_{E_{0}}\right)\right)$

$<G\left(\eta\left(\left\|\phi_{n+1}-\phi_{n+2}\right\|_{E_{0}}\right), \alpha\left(\phi_{n}(c), \phi_{n+1}(c)\right) \eta\left(\left\|\phi_{n+1}-\phi_{n+2}\right\|_{E_{0}}\right)\right)$. (by $\left.\left(\zeta_{8}\right)\right)$

Now by the property $C_{G}$ and (36), we get

$\eta\left(\left\|\phi_{n+1}-\phi_{n+2}\right\|_{E_{0}}\right)>\alpha\left(\phi_{n}(c), \phi_{n+1}(c)\right) \eta\left(\left\|\phi_{n+1}-\phi_{n+2}\right\|_{E_{0}}\right)$

a contradiction.

$$
\geq \eta\left(\left\|\phi_{n+1}-\phi_{n+2}\right\|_{E_{0}}\right) \text {, }
$$

Therefore

$M\left(\phi_{n}, \phi_{n+1}\right)=\left\|\phi_{n}-\phi_{n+1}\right\|_{E_{0}}$ and hence $\left\|\phi_{n+1}-\phi_{n+2}\right\|_{E_{0}}<\left\|\phi_{n}-\phi_{n+1}\right\|_{E_{0}}$.

Therefore the sequence $\left\{\left\|\phi_{n}-\phi_{n+1}\right\|_{E_{0}}\right\}$ is a monotonically decreasing sequence in $\mathbb{R}^{+}$and hence it is convergent.

Let $\lim _{n \rightarrow \infty}\left\|\phi_{n}-\phi_{n+1}\right\|_{E_{0}}=k$ (say). Suppose that $k>0$.

Clearly $\eta\left(\left\|\phi_{n}-\phi_{n+1}\right\|_{E_{0}}\right)>0$.

From (38), we have

$$
\begin{aligned}
C_{G} & \leq \zeta\left(\alpha\left(\phi_{n}(c), \phi_{n+1}(c)\right) \eta\left(\left\|T \phi_{n}-T \phi_{n+1}\right\|_{E}\right), \eta\left(M\left(\phi_{n}, \phi_{n+1}\right)\right)\right) \\
& =\zeta\left(\alpha\left(\phi_{n}(c), \phi_{n+1}(c)\right) \eta\left(\left\|T \phi_{n}-T \phi_{n+1}\right\|_{E}\right), \eta\left(\left\|\phi_{n}-\phi_{n+1}\right\|_{E_{0}}\right)\right) \\
& <G\left(\eta\left(\left\|\phi_{n}-\phi_{n+1}\right\|_{E_{0}}\right), \alpha\left(\phi_{n}(c), \phi_{n+1}(c)\right) \eta\left(\left\|T \phi_{n}-T \phi_{n+1}\right\|_{E}\right)\right) .
\end{aligned}
$$

Now by the property $C_{G}$, we get

$$
\begin{aligned}
& \eta\left(\left\|\phi_{n}-\phi_{n+1}\right\|_{E_{0}}\right)>\alpha\left(\phi_{n}(c), \phi_{n+1}(c)\right) \eta\left(\left\|T \phi_{n}-T \phi_{n+1}\right\|_{E}\right) \\
& \quad=\alpha\left(\phi_{n}(c), \phi_{n+1}(c)\right) \eta\left(\left\|\phi_{n+1}-\phi_{n+2}\right\|_{E_{0}}\right) \\
& \quad \geq \eta\left(\left\|\phi_{n+1}-\phi_{n+2}\right\|_{E_{0}}\right) .
\end{aligned}
$$

On applying limits as $n \rightarrow \infty$, we get

$\eta(k) \geq \lim _{n \rightarrow \infty} \alpha\left(\phi_{n}(c), \phi_{n+1}(c)\right) \eta\left(\left\|\phi_{n+1}-\phi_{n+2}\right\|_{E_{0}}\right) \geq \eta(k)$.

Therefore

$$
\lim _{n \rightarrow \infty} \alpha\left(\phi_{n}(c), \phi_{n+1}(c)\right) \eta\left(\left\|\phi_{n+1}-\phi_{n+2}\right\|_{E_{0}}\right)=\eta(k)>0 .
$$


Clearly $\lim _{n \rightarrow \infty} M\left(\phi_{n}, \phi_{n+1}\right)=k$.

Since $\eta$ is continuous we have

$$
\lim _{n \rightarrow \infty} \eta\left(M\left(\phi_{n}, \phi_{n+1}\right)\right)=\eta(k)>0 .
$$

On applying limit superior as $n \rightarrow \infty$ to 38 , we get

$$
\begin{aligned}
C_{G} & \leq \limsup _{n \rightarrow \infty} \zeta\left(\alpha\left(\phi_{n}(c), \phi_{n+1}(c)\right) \eta\left(\left\|T \phi_{n}-T \phi_{n+1}\right\|_{E}\right), \eta\left(M\left(\phi_{n}, \phi_{n+1}\right)\right)\right) \\
& =\limsup _{n \rightarrow \infty} \zeta\left(\alpha\left(\phi_{n}(c), \phi_{n+1}(c)\right) \eta\left(\left\|\phi_{n+1}-\phi_{n+2}\right\|_{E_{0}}\right), \eta\left(\left\|\phi_{n}-\phi_{n+1}\right\|_{E_{0}}\right)\right) \\
& <C_{G}\left(\text { from 39, } 40, \text { 41) and }\left(\zeta_{9}\right)\right),
\end{aligned}
$$

a contradiction.

Therefore $k=0$ and hence

$$
\lim _{n \rightarrow \infty}\left\|\phi_{n}-\phi_{n+1}\right\|_{E_{0}}=0 .
$$

We now show that the sequence $\left\{\phi_{n}\right\}$ is a Cauchy sequence in $R_{c}$.

Suppose that the sequence $\left\{\phi_{n}\right\}$ is not a Cauchy sequence.

Then there exists an $\epsilon>0$ and two subsequences $\left\{\phi_{m_{k}}\right\}$ and $\left\{\phi_{n_{k}}\right\}$ of $\left\{\phi_{n}\right\}$ with $m_{k}>n_{k}>k$ such that $\left\|\phi_{n_{k}}-\phi_{m_{k}}\right\|_{E_{0}} \geq \epsilon,\left\|\phi_{n_{k}}-\phi_{m_{k}-1}\right\|_{E_{0}}<\epsilon$ and from Lemma 1.34 we have

$$
\lim _{k \rightarrow \infty}\left\|\phi_{n_{k}}-\phi_{m_{k}}\right\|_{E_{0}}=\epsilon
$$

and

$\lim _{k \rightarrow \infty}\left\|\phi_{n_{k}}-\phi_{m_{k}+1}\right\|_{E_{0}}=\epsilon=\lim _{k \rightarrow \infty}\left\|\phi_{n_{k}+1}-\phi_{m_{k}}\right\|_{E_{0}}$

Since $\eta$ is continuous, we get

$$
=\lim _{k \rightarrow \infty}\left\|\phi_{n_{k}+1}-\phi_{m_{k}+1}\right\|_{E_{0}} \text {. }
$$

$$
\lim _{k \rightarrow \infty} \eta\left(\left\|\phi_{n_{k}+1}-\phi_{m_{k}+1}\right\|_{E_{0}}\right)=\eta(\epsilon)>0
$$

We consider

$$
\begin{aligned}
& M\left(\phi_{n_{k}}, \phi_{m_{k}}\right)=\max \left\{\left\|\phi_{n_{k}}-\phi_{m_{k}}\right\|_{E_{0}},\left\|\phi_{n_{k}}(c)-T \phi_{n_{k}}\right\|_{E},\left\|\phi_{m_{k}}(c)-T \phi_{m_{k}}\right\|_{E},\right. \\
& \left.\frac{\left\|\phi_{n_{k}}(c)-T \phi_{m_{k}}\right\|_{E}+\left\|\phi_{m_{k}}(c)-T \phi_{n_{k}}\right\|_{E}}{2}\right\} \\
& =\max \left\{\left\|\phi_{n_{k}}-\phi_{m_{k}}\right\|_{E_{0}},\left\|\phi_{n_{k}}-\phi_{n_{k}+1}\right\|_{E_{0}}^{2},\left\|\phi_{m_{k}}-\phi_{m_{k}+1}\right\|_{E_{0}}\right. \text {, } \\
& \left.\frac{\left\|\phi_{n_{k}}-\phi_{m_{k}+1}\right\|_{E_{0}}+\left\|\phi_{m_{k}}-\phi_{n_{k}+1}\right\|_{E_{0}}}{2}\right\} \text {. }
\end{aligned}
$$

On applying limits as $k \rightarrow \infty$, we get

$\lim _{k \rightarrow \infty} M\left(\phi_{n_{k}}, \phi_{m_{k}}\right)=\epsilon$ and hence

$$
\lim _{k \rightarrow \infty} \eta\left(M\left(\phi_{n_{k}}, \phi_{m_{k}}\right)\right)=\eta(\epsilon)>0 .
$$

From (44) and (45), there exists $k_{1} \in \mathbb{N}$ such that

$$
\eta\left(M\left(\phi_{n_{k}}, \phi_{m_{k}}\right)\right)>\frac{\eta(\epsilon)}{2}>0 \text { for any } k \geq k_{1}
$$

and

$\eta\left(\left\|T \phi_{n_{k}}-T \phi_{m_{k}}\right\|_{E}\right)=\eta\left(\left\|\phi_{n_{k}+1}-\phi_{m_{k}+1}\right\|_{E_{0}}\right)>\frac{\eta(\epsilon)}{2}>0$ for any $k \geq k_{1}$.

From $(36)$, we get

$$
\alpha\left(\phi_{n_{k}}(c), \phi_{m_{k}}(c)\right) \eta\left(\left\|T \phi_{n_{k}}-T \phi_{m_{k}}\right\|_{E}\right) \geq \eta\left(\left\|T \phi_{n_{k}}-T \phi_{m_{k}}\right\|_{E}\right)>0
$$

for any $k \geq k_{1}$.

Suppose that there exists $k \geq k_{1}$ such that $\left\|\phi_{n_{k}}-\phi_{n_{k}+1}\right\|_{E_{0}}>\left\|\phi_{n_{k}}-\phi_{m_{k}}\right\|_{E_{0}}$.

On applying limits as $k \rightarrow \infty$, we get $0 \geq \epsilon$, a contradiction.

Therefore

$$
\left\|\phi_{n_{k}}-\phi_{n_{k}+1}\right\|_{E_{0}} \leq\left\|\phi_{n_{k}}-\phi_{m_{k}}\right\|_{E_{0}}
$$


for any $k \geq k_{1}$.

Now for any $k \geq k_{1}$, we have

$$
\begin{aligned}
\frac{1}{2} \mu\left(\phi_{n_{k}}(c), \phi_{m_{k}}(c)\right)\left\|\phi_{n_{k}}(c)-T \phi_{n_{k}}\right\|_{E} & \leq \frac{1}{2}\left\|\phi_{n_{k}}-\phi_{n_{k}+1}\right\|_{E_{0}}\left(\text { since } n_{k}<m_{k}\right) \\
& \leq \frac{1}{2}\left\|\phi_{n_{k}}-\phi_{m_{k}}\right\|_{E_{0}} \\
& <\left\|\phi_{n_{k}}-\phi_{m_{k}}\right\|_{E_{0}} .
\end{aligned}
$$

From (33), we get

$$
\begin{gathered}
C_{G} \leq \zeta\left(\alpha\left(\phi_{n_{k}}(c), \phi_{m_{k}}(c)\right) \eta\left(\left\|T \phi_{n_{k}}-T \phi_{m_{k}}\right\|_{E}\right), \eta\left(M\left(\phi_{n_{k}}, \phi_{m_{k}}\right)\right)\right) \\
<G\left(\eta\left(M\left(\phi_{n_{k}}, \phi_{m_{k}}\right)\right), \alpha\left(\phi_{n_{k}}(c), \phi_{m_{k}}(c)\right) \eta\left(\left\|T \phi_{n_{k}}-T \phi_{m_{k}}\right\|_{E}\right)\right) . \\
\text { (from } \left.46, \text { 477) and }\left(\zeta_{8}\right)\right)
\end{gathered}
$$

Now by the property $C_{G}$, we get

On applying limits as $k \rightarrow \infty$, we get

$$
\begin{gathered}
\eta\left(M\left(\phi_{n_{k}}, \phi_{m_{k}}\right)\right)>\alpha\left(\phi_{n_{k}}(c), \phi_{m_{k}}(c)\right) \eta\left(\left\|T \phi_{n_{k}}-T \phi_{m_{k}}\right\|_{E}\right) \\
\geq \eta\left(\left\|T \phi_{n_{k}}-T \phi_{m_{k}}\right\|_{E}\right)=\eta\left(\left\|\phi_{n_{k}+1}-\phi_{m_{k}+1}\right\|_{E_{0}}\right) .
\end{gathered}
$$

$$
\lim _{k \rightarrow \infty} \alpha\left(\phi_{n_{k}}(c), \phi_{m_{k}}(c)\right) \eta\left(\left\|T \phi_{n_{k}}-T \phi_{m_{k}}\right\|_{E}\right)=\eta(\epsilon)>0 .
$$

On applying limit superior as $k \rightarrow \infty$ to $(49)$, by (50) and $\left(\zeta_{9}\right)$ we get

$C_{G} \leq \limsup _{k \rightarrow \infty} \zeta\left(\alpha\left(\phi_{n_{k}}(c), \phi_{m_{k}}(c)\right) \eta\left(\left\|T \phi_{n_{k}}-T \phi_{m_{k}}\right\|_{E}\right), \eta\left(M\left(\phi_{n_{k}}, \phi_{m_{k}}\right)\right)\right)$

$<C_{G}$,

a contradiction.

Therefore the sequence $\left\{\phi_{n}\right\}$ is a Cauchy sequence in $R_{c}$.

Since $E_{0}$ is complete, there exists $\phi^{*} \in E_{0}$ such that $\phi_{n} \rightarrow \phi^{*}$ as $n \rightarrow \infty$.

Since $R_{c}$ is topologically closed, we have $\phi^{*} \in R_{c}$.

We now show that $T \phi^{*}=\phi^{*}(c)$. Suppose that $T \phi^{*} \neq \phi^{*}(c)$.

From (36), we have $\alpha\left(\phi_{n}(c), \phi_{n+1}(c)\right) \geq 1$ and $\mu\left(\phi_{n}(c), \phi_{n+1}(c)\right) \leq 1$

for any $n \in \mathbb{N} \cup\{0\}$. From (iv), we get

$\alpha\left(\phi_{n}(c), \phi^{*}(c)\right) \geq 1$ and $\mu\left(\phi_{n}(c), \phi^{*}(c)\right) \leq 1$ for any $n \in \mathbb{N} \cup\{0\}$.

First we show that either

$$
\frac{1}{2} \mu\left(\phi_{n}(c), \phi^{*}(c)\right)\left\|\phi_{n}(c)-T \phi_{n}\right\|_{E}<\left\|\phi_{n}-\phi^{*}\right\|_{E_{0}}
$$

or

$$
\frac{1}{2} \mu\left(\phi_{n+1}(c), \phi^{*}(c)\right)\left\|\phi_{n+1}(c)-T \phi_{n+1}\right\|_{E}<\left\|\phi_{n+1}-\phi^{*}\right\|_{E_{0}} \text { holds }
$$

for any $n \in \mathbb{N} \cup\{0\}$.

Suppose that there exists $m \in \mathbb{N} \cup\{0\}$ such that

$$
\frac{1}{2} \mu\left(\phi_{m}(c), \phi^{*}(c)\right)\left\|\phi_{m}(c)-T \phi_{m}\right\|_{E} \geq\left\|\phi_{m}-\phi^{*}\right\|_{E_{0}}
$$

and

$$
\frac{1}{2} \mu\left(\phi_{m+1}(c), \phi^{*}(c)\right)\left\|\phi_{m+1}(c)-T \phi_{m+1}\right\|_{E} \geq\left\|\phi_{m+1}-\phi^{*}\right\|_{E_{0}} .
$$

From (52), we have

$\left\|\phi_{m}-\phi^{*}\right\|_{E_{0}} \leq \frac{1}{2} \mu\left(\phi_{m}(c), \phi^{*}(c)\right)\left\|\phi_{m}(c)-T \phi_{m}\right\|_{E}$

Therefore

$$
\leq \frac{1}{2}\left\|\phi_{m}(c)-T \phi_{m}\right\|_{E} \text {. }
$$

$2\left\|\phi_{m}-\phi^{*}\right\|_{E_{0}} \leq\left\|\phi_{m}(c)-\phi^{*}(c)\right\|_{E}+\left\|\phi^{*}(c)-T \phi_{m}\right\|_{E}$

and hence

$$
=\left\|\phi_{m}-\phi^{*}\right\|_{E_{0}}+\left\|\phi^{*}-\phi_{m+1}\right\|_{E_{0}}
$$

$\left\|\phi_{m}-\left.\phi^{*}\right|_{E_{0}} \leq\right\| \phi_{m+1}-\phi^{*} \|_{E_{0}}$ 
Clearly

$$
\begin{aligned}
& \leq \frac{1}{2} \mu\left(\phi_{m+1}(c), \phi^{*}(c)\right)\left\|\phi_{m+1}(c)-T \phi_{m+1}\right\|_{E}(\text { by } 53 \\
& \leq \frac{1}{2}\left\|\phi_{m+1}-\phi_{m+2}\right\|_{E_{0}} .
\end{aligned}
$$

$\left\|\phi_{m+1}-\phi_{m+2}\right\|_{E_{0}}<\left\|\phi_{m}-\phi_{m+1}\right\|_{E_{0}}$

$$
\begin{aligned}
& \leq\left\|\phi_{m}-\phi^{*}\right\|_{E_{0}}+\left\|\phi^{*}-\phi_{m+1}\right\|_{E_{0}} \\
& \leq \frac{1}{2}\left\|\phi_{m+1}-\phi_{m+2}\right\|_{E_{0}}+\frac{1}{2}\left\|\phi_{m+1}-\phi_{m+2}\right\|_{E_{0}} \\
& =\left\|\phi_{m+1}-\phi_{m+2}\right\|_{E_{0}},
\end{aligned}
$$

a contradiction.

Therefore either

$$
\frac{1}{2} \mu\left(\phi_{n}(c), \phi^{*}(c)\right)\left\|\phi_{n}(c)-T \phi_{n}\right\|_{E}<\left\|\phi_{n}-\phi^{*}\right\|_{E_{0}}
$$

or

$$
\frac{1}{2} \mu\left(\phi_{n+1}(c), \phi^{*}(c)\right)\left\|\phi_{n+1}(c)-T \phi_{n+1}\right\|_{E}<\left\|\phi_{n+1}-\phi^{*}\right\|_{E_{0}}
$$

holds for any $n \in \mathbb{N} \cup\{0\}$.

Case (i): Suppose that $\frac{1}{2} \mu\left(\phi_{n}(c), \phi^{*}(c)\right)\left\|\phi_{n}(c)-T \phi_{n}\right\|_{E}<\left\|\phi_{n}-\phi^{*}\right\|_{E_{0}}$.

From (33), we get

$$
C_{G} \leq \zeta\left(\alpha\left(\phi_{n}(c), \phi^{*}(c)\right) \eta\left(\left\|T \phi_{n}-T \phi^{*}\right\|_{E_{0}}\right), \eta\left(M\left(\phi_{n}, \phi^{*}\right)\right)\right) .
$$

We consider

$$
\begin{aligned}
& M\left(\phi_{n}, \phi^{*}\right)=\max \left\{\left\|\phi_{n}-\phi^{*}\right\|_{E_{0}},\left\|\phi_{n}(c)-T \phi_{n}\right\|_{E_{0}},\left\|\phi^{*}(c)-T \phi^{*}\right\|_{E},\right. \\
& \left.\frac{\left\|\phi_{n}(c)-T \phi^{*}\right\|_{E}+\left\|\phi^{*}(c)-T \phi_{n}\right\|_{E}}{2}\right\} .
\end{aligned}
$$

If $M\left(\phi_{n}, \phi^{*}\right)=0$ then $T \phi^{*}=\phi^{*}(c)$, a contradiction.

Therefore $M\left(\phi_{n}, \phi^{*}\right)>0$ and hence $\eta\left(M\left(\phi_{n}, \phi^{*}\right)\right)>0$.

If $\eta\left(\left\|T \phi_{n}-T \phi^{*}\right\|_{E_{0}}\right)=0$ then $T \phi_{n}=T \phi^{*}$ and hence $T \phi^{*}=\phi_{n+1}(c)$.

On applying limits as $n \rightarrow \infty$, we get $T \phi^{*}=\phi^{*}(c)$, a contradiction.

Therefore $\eta\left(\left\|T \phi_{n}-T \phi^{*}\right\|_{E}\right)>0$ and hence

$\alpha\left(\phi_{n}(c), \phi^{*}(c)\right) \eta\left(\left\|T \phi_{n}-T \phi^{*}\right\|_{E}\right)>0$.

On applying limits to $M\left(\phi_{n}, \phi^{*}\right)$ as $n \rightarrow \infty$, we get

$\lim _{n \rightarrow \infty} M\left(\phi_{n}, \phi^{*}\right)=\left\|\phi^{*}(c)-T \phi^{*}\right\|_{E}$.

Since $\eta$ is continuous, we have

$$
\lim _{n \rightarrow \infty} \eta\left(M\left(\phi_{n}, \phi^{*}\right)\right)=\eta\left(\left\|\phi^{*}(c)-T \phi^{*}\right\|_{E}\right)>0 .
$$

From (54), we have

$C_{G} \leq \zeta\left(\alpha\left(\phi_{n}(c), \phi^{*}(c)\right) \eta\left(\left\|T \phi_{n}-T \phi^{*}\right\|_{E}\right), \eta\left(M\left(\phi_{n}, \phi^{*}\right)\right)\right)$

$$
\left.<G\left(\eta\left(M\left(\phi_{n}, \phi^{*}\right)\right), \alpha\left(\phi_{n}(c), \phi^{*}(c)\right) \eta\left(\left\|T \phi_{n}-T \phi^{*}\right\|_{E}\right)\right) \text {. (by }\left(\zeta_{8}\right)\right)
$$

Now by the property $C_{G}$, we get

$$
\begin{aligned}
& \eta\left(M\left(\phi_{n}, \phi^{*}\right)\right)>\alpha\left(\phi_{n}(c), \phi^{*}(c)\right) \eta\left(\left\|T \phi_{n}-T \phi^{*}\right\|_{E}\right) \\
\geq & \eta\left(\left\|T \phi_{n}-T \phi^{*}\right\|_{E}\right)=\eta\left(\left\|\phi_{n+1}(c)-T \phi^{*}\right\|_{E}\right) .
\end{aligned}
$$

On applying limits as $n \rightarrow \infty$, we get

$$
\lim _{n \rightarrow \infty} \alpha\left(\phi_{n}(c), \phi^{*}(c)\right) \eta\left(\left\|T \phi_{n}-T \phi^{*}\right\|_{E}\right)=\eta\left(\left\|\phi^{*}(c)-T \phi^{*}\right\|_{E}\right)>0 .
$$

On applying limit superior as $n \rightarrow \infty$ to (54), by (56) and $\left(\zeta_{9}\right)$ we get $C_{G} \leq \limsup _{n \rightarrow \infty} \zeta\left(\alpha\left(\phi_{n}(c), \phi^{*}(c)\right) \eta\left(\left\|T \phi_{n}-T \phi^{*}\right\|_{E}\right), \eta\left(M\left(\phi_{n}, \phi^{*}\right)\right)\right)<C_{G}$, a contradiction.

Case (ii): Suppose that

From (33), we get

$$
\frac{1}{2} \mu\left(\phi_{n+1}(c), \phi^{*}(c)\right)\left\|\phi_{n+1}(c)-T \phi_{n+1}\right\|_{E}<\left\|\phi_{n+1}-\phi^{*}\right\|_{E_{0}} .
$$

$$
C_{G} \leq \zeta\left(\alpha\left(\phi_{n+1}(c), \phi^{*}(c)\right) \eta\left(\left\|T \phi_{n+1}-T \phi^{*}\right\|_{E_{0}}\right), \eta\left(M\left(\phi_{n+1}, \phi^{*}\right)\right)\right)
$$


We consider

$$
M\left(\phi_{n+1}, \phi^{*}\right)=\max \left\{\left\|\phi_{n+1}-\phi^{*}\right\|_{E_{0}},\left\|\phi_{n+1}(c)-T \phi_{n+1}\right\|_{E_{0}},\left\|\phi^{*}(c)-T \phi^{*}\right\|_{E},\right.
$$

If $M\left(\phi_{n+1}, \phi^{*}\right)=0$ then $T \phi^{*}=\phi^{*}(c)$, a contradiction.

Therefore $M\left(\phi_{n+1}, \phi^{*}\right)>0$ and hence $\eta\left(M\left(\phi_{n+1}, \phi^{*}\right)\right)>0$.

If $\eta\left(\left\|T \phi_{n+1}-T \phi^{*}\right\|_{E}\right)=0$ then $T \phi_{n+1}=T \phi^{*}$ and hence $\phi_{n+2}(c)=T \phi^{*}$.

On applying limits as $n \rightarrow \infty$, we get $T \phi^{*}=\phi^{*}(c)$, a contradiction.

Therefore $\eta\left(\left\|T \phi_{n+1}-T \phi^{*}\right\|_{E}\right)>0$ and hence

$\alpha\left(\phi_{n+1}(c), \phi^{*}(c)\right) \eta\left(\left\|T \phi_{n+1}-T \phi^{*}\right\|_{E}\right)>0$

On applying limits to $M\left(\phi_{n+1}, \phi^{*}\right)$ as $n \rightarrow \infty$, we get

$\lim _{n \rightarrow \infty} M\left(\phi_{n+1}, \phi^{*}\right)=\left\|\phi^{*}(c)-T \phi^{*}\right\|_{E}$.

Since $\eta$ is continuous, we have

$$
\lim _{n \rightarrow \infty} \eta\left(M\left(\phi_{n+1}, \phi^{*}\right)\right)=\eta\left(\left\|\phi^{*}(c)-T \phi^{*}\right\|_{E}\right)>0 .
$$

From (58), we have

$C_{G} \leq \zeta\left(\alpha\left(\phi_{n+1}(c), \phi^{*}(c)\right) \eta\left(\left\|T \phi_{n+1}-T \phi^{*}\right\|_{E}\right), \eta\left(M\left(\phi_{n+1}, \phi^{*}\right)\right)\right)$

$<G\left(\eta\left(M\left(\phi_{n+1}, \phi^{*}\right)\right), \alpha\left(\phi_{n+1}(c), \phi^{*}(c)\right) \eta\left(\left\|T \phi_{n+1}-T \phi^{*}\right\|_{E}\right)\right)$. (by $\left.\left(\zeta_{8}\right)\right)$

Now by the property $C_{G}$, we get

$$
\begin{aligned}
& \eta\left(M\left(\phi_{n+1}, \phi^{*}\right)\right)>\alpha\left(\phi_{n+1}(c), \phi^{*}(c)\right) \eta\left(\left\|T \phi_{n+1}-T \phi^{*}\right\|_{E}\right) \\
& \geq \eta\left(\left\|T \phi_{n+1}-T \phi^{*}\right\|_{E}\right)=\eta\left(\left\|\phi_{n+2}(c)-T \phi^{*}\right\|_{E}\right) .
\end{aligned}
$$

On applying limits as $n \rightarrow \infty$, we get

$$
\lim _{n \rightarrow \infty} \alpha\left(\phi_{n+1}(c), \phi^{*}(c)\right) \eta\left(\left\|T \phi_{n+1}-T \phi^{*}\right\|_{E}\right)=\eta\left(\left\|\phi^{*}(c)-T \phi^{*}\right\|_{E}\right)>0 .
$$

On applying limit superior to (58) as $n \rightarrow \infty$, by (60) and $\left(\zeta_{9}\right)$ we get

$C_{G} \leq \limsup _{n \rightarrow \infty} \zeta\left(\alpha\left(\phi_{n+1}(c), \phi^{*}(c)\right) \eta\left(\left\|T \phi_{n+1}-T \phi^{*}\right\|_{E}\right), \eta\left(M\left(\phi_{n+1}, \phi^{*}\right)\right)\right)$

$<C_{G}^{n \rightarrow}$

a contradiction.

Therefore from Case(i) and Case (ii), we conclude that $T \phi^{*}=\phi^{*}(c)$ and hence $\phi^{*} \in R_{c}$ is a PPF dependent fixed point of $T$.

Suppose that $T$ is one-one, $\alpha(x, y) \geq 1$ and $\mu(x, y) \leq 1$ for any $x, y \in E$.

We now show that $T$ has a unique PPF dependent fixed point in $R_{c}$.

Let $\phi, \psi \in R_{c}$ be two PPF dependent fixed points of $T$.

Then $T \phi=\phi(c)$ and $T \psi=\psi(c)$.

Since $R_{c}$ is algebraically closed with respect to the difference, we have $\|\phi-\psi\|_{E_{0}}=\|\phi(c)-\psi(c)\|_{E}$. Suppose that $\phi \neq \psi$.

If $\|T \phi-T \psi\|_{E}=0$ then $T \phi=T \psi$.

Since $T$ is one-one we have $\phi=\psi$, a contradiction.

Therefore $\|T \phi-T \psi\|_{E} \neq 0$ and hence $\|T \phi-T \psi\|_{E}>0$.

Clearly $\eta\left(\|T \phi-T \psi\|_{E}\right)>0$ and hence $\alpha(\phi(c), \psi(c)) \eta\left(\|T \phi-T \psi\|_{E}\right)>0$.

Clearly $0=\frac{1}{2} \mu(\phi(c), \psi(c))\|\phi(c)-T \phi\|_{E}<\|\phi-\psi\|_{E_{0}}$.

From (33), we get

$$
C_{G} \leq \zeta\left(\alpha(\phi(c), \psi(c)) \eta\left(\|T \phi-T \psi\|_{E}\right), \eta(M(\phi, \psi))\right) .
$$

We consider

$$
\begin{aligned}
M(\phi, \psi) & =\max \left\{\|\phi-\psi\|_{E_{0}},\|\phi(c)-T \phi\|_{E},\|\psi(c)-T \psi\|_{E},\right. \\
& =\max \left\{\|\phi-\psi\|_{E_{0}}, \frac{\|\phi(c)-T \psi(c)\|_{E}+\|\psi(c)-\phi(c)\| \|_{E}}{2}\right\}
\end{aligned}
$$


$\eta(M(\phi, \psi))>0$.

$$
=\max \left\{\|\phi-\psi\|_{E_{0}},\|\phi-\psi\|_{E_{0}}\right\}=\|\phi-\psi\|_{E_{0}} \text { and hence }
$$

By $(62)$ and $\left(\zeta_{8}\right)$, we get

$C_{G}<G\left(\eta(M(\phi, \psi)), \alpha(\phi(c), \psi(c)) \eta\left(\|T \phi-T \psi\|_{E}\right)\right)$.

Now by the property $C_{G}$, we get $\eta(M(\phi, \psi))>\alpha(\phi(c), \psi(c)) \eta\left(\|T \phi-T \psi\|_{E}\right) \geq \eta\left(\|T \phi-T \psi\|_{E}\right)$ and which implies that $\eta\left(\|\phi-\psi\|_{E_{0}}\right)>$ $\eta\left(\|T \phi-T \psi\|_{E}\right)$

a contradiction.

$$
\begin{aligned}
& =\eta\left(\|\phi(c)-\psi(c)\|_{E}\right) \\
& =\eta\left(\|\phi-\psi\|_{E_{0}}\right),
\end{aligned}
$$

Therefore $\phi=\psi$ and hence $T$ has a unique PPF dependent fixed point in $R_{c}$.

\section{Corollaries and Examples}

Corollary 4.1. Let $c \in I$. Let $T: E_{0} \rightarrow E$ be a function satisfying the following conditions:

(i) $T$ is a generalized Suzuki type $Z_{G, \alpha, \eta}$-contraction with respect to $\zeta$,

(ii) $R_{c}$ is algebraically closed with respect to the difference,

(iii) $T$ is a triangular $\alpha_{c}-$ admissible mapping,

(iv) there exists $\phi_{0} \in R_{c}$ such that $\alpha\left(\phi_{0}(c), T \phi_{0}\right) \geq 1$ and

(v) if $\left\{\phi_{n}\right\}$ is a sequence in $E_{0}$ such that $\phi_{n} \rightarrow \phi$ as $n \rightarrow \infty$,

$\alpha\left(\phi_{n}(c), \phi_{n+1}(c)\right) \geq 1$ for any $n \in \mathbb{N} \cup\{0\}$ then $\alpha\left(\phi_{n}(c), \phi(c)\right) \geq 1$

for any $n \in \mathbb{N} \cup\{0\}$.

Then $T$ has a PPF dependent fixed point in $R_{c}$. Moreover, if $T$ is one-one and $\alpha(x, y) \geq 1$ for any $x, y \in E$ then $T$ has a unique PPF dependent fixed point in $R_{c}$.

Proof. By taking $\mu(x, y)=1$ for any $x, y \in E$ in Theorem 3.1 we obtain the desired result.

Corollary 4.2. Let $c \in I$. Let $T: E_{0} \rightarrow E$ be a function satisfying the following conditions:

(i) $T$ is a generalized Suzuki type $Z_{G, \eta}$-contraction with respect to $\zeta$,

(ii) $R_{c}$ is algebraically closed with respect to the difference.

Then $T$ has a PPF dependent fixed point in $R_{c}$. Moreover, if $T$ is one-one then $T$ has a unique PPF dependent fixed point in $R_{c}$.

Proof. By taking $\alpha(x, y)=1$ for any $x, y \in E$ in Corollary 4.1 we obtain the desired result.

Corollary 4.3. Let $c \in I$. Let $T: E_{0} \rightarrow E$ be a function satisfying the following conditions:

(i) $T$ is a generalized Suzuki type $Z_{H, \alpha, \eta}$-contraction with respect to $\zeta$,

(ii) $R_{c}$ is algebraically closed with respect to the difference,

(iii) $T$ is a triangular $\alpha_{c}-$ admissible mapping,

(iv) there exists $\phi_{0} \in R_{c}$ such that $\alpha\left(\phi_{0}(c), T \phi_{0}\right) \geq 1$ and

(v) if $\left\{\phi_{n}\right\}$ is a sequence in $E_{0}$ such that $\phi_{n} \rightarrow \phi$ as $n \rightarrow \infty$, $\alpha\left(\phi_{n}(c), \phi_{n+1}(c)\right) \geq 1$ for any $n \in \mathbb{N} \cup\{0\}$ then $\alpha\left(\phi_{n}(c), \phi(c)\right) \geq 1$

for any $n \in \mathbb{N} \cup\{0\}$.

Then $T$ has a PPF dependent fixed point in $R_{c}$. Moreover, if $T$ is one-one and $\alpha(x, y) \geq 1$ for any $x, y \in E$ then $T$ has a unique PPF dependent fixed point in $R_{c}$.

Proof. By taking $\mu(x, y)=1$ for any $x, y \in E, G(s, t)=s-t$ for any $s, t \in \mathbb{R}^{+}$and $C_{G}=0$ in Theorem 3.1 we obtain the desired result.

Corollary 4.4. Let $c \in I$. Let $T: E_{0} \rightarrow E$ be a function satisfying the following conditions:

(i) $T$ is a generalized Suzuki type $Z_{H, \alpha}$-contraction with respect to $\zeta$,

(ii) $R_{c}$ is algebraically closed with respect to the difference and 
(iii) $T$ is a triangular $\alpha_{c}-$ admissible mapping,

(iv) there exists $\phi_{0} \in R_{c}$ such that $\alpha\left(\phi_{0}(c), T \phi_{0}\right) \geq 1$ and

(v) if $\left\{\phi_{n}\right\}$ is a sequence in $E_{0}$ such that $\phi_{n} \rightarrow \phi$ as $n \rightarrow \infty$,

$\alpha\left(\phi_{n}(c), \phi_{n+1}(c)\right) \geq 1$ for any $n \in \mathbb{N} \cup\{0\}$ then $\alpha\left(\phi_{n}(c), \phi(c)\right) \geq 1$

for any $n \in \mathbb{N} \cup\{0\}$.

Then $T$ has a PPF dependent fixed point in $R_{c}$. Moreover, if $T$ is one-one and $\alpha(x, y) \geq 1$ for any $x, y \in E$ then $T$ has a unique PPF dependent fixed point in $R_{c}$.

Proof. By taking $\eta=$ Identity mapping in Corollary 4.3 we obtain the desired result.

Corollary 4.5. Let $c \in I$. Let $T: E_{0} \rightarrow E$ be a function satisfying the following conditions:

(i) $T$ is a generalized Suzuki type $Z_{H}$-contraction with respect to $\zeta$,

(ii) $R_{c}$ is algebraically closed with respect to the difference.

Then $T$ has a PPF dependent fixed point in $R_{c}$. Moreover, if $T$ is one-one then $T$ has a unique PPF dependent fixed point in $R_{c}$.

Proof. By taking $\alpha(x, y)=1$ for any $x, y \in E$ in Corollary 4.4 we obtain the desired result.

Example 4.6. Let $E=\mathbb{R}, c=1 \in I=\left[\frac{1}{2}, 2\right] \subseteq \mathbb{R}, E_{0}=C(I, E)$.

We define $T: E_{0} \rightarrow E$ by

$$
T \phi= \begin{cases}\frac{\phi(c)}{16} & \text { if } 0 \leq \phi(c) \leq 1 \\ \frac{\phi(c)}{8} & \text { if otherwise, }\end{cases}
$$

for any $\phi \in E_{0}$.

We define $\eta: \mathbb{R}^{+} \rightarrow \mathbb{R}^{+}$by $\eta(x)=2 x$ for any $x \in \mathbb{R}^{+}$. Clearly $\eta \in \Psi$.

We define $\zeta: \mathbb{R}^{+} \times \mathbb{R}^{+} \rightarrow \mathbb{R}$ by $\zeta(t, s)=\lambda s-t$, where $\lambda \in(0,1), C_{G}=0$ and

$G: \mathbb{R}^{+} \times \mathbb{R}^{+} \rightarrow \mathbb{R}$ by $G(s, t)=s-t$ for any $s, t \in \mathbb{R}^{+}$.

Clearly $\zeta \in Z_{G}$. (Example 1.20).

Let $\phi, \psi \in E_{0}$ be such that $\phi \neq \psi$.

Assume that

$$
\frac{1}{2}\|\phi(c)-T \phi\|_{E}<\|\phi-\psi\|_{E_{0}} .
$$

Case (i): Suppose that $T \phi=\frac{\phi(c)}{16}$ and $T \psi=\frac{\psi(c)}{16}$.

$\overline{\text { Clearly } \|} \boldsymbol{\|} \phi-T \psi\left\|_{E}=\frac{1}{16}\right\| \phi(c)-\psi(c) \|_{E}$ which implies that

$\eta\left(\|T \phi-T \psi\|_{E}\right)=\eta\left(\frac{1}{16}\|\phi(c)-\psi(c)\|_{E}\right)=\frac{1}{8}\|\phi(c)-\psi(c)\|_{E}$

$$
\begin{aligned}
& \leq \frac{1}{8}\left[\|\phi(c)-T \phi\| \|_{E}\right]+\frac{1}{8}\left[\|T \phi-\psi(c)\| \|_{E}\right] \\
& <\frac{1}{4}\|\phi-\psi\|_{E_{0}}+\frac{1}{8}\left[\|T \phi-\psi(c)\|_{E}\right](b y[63) \\
& \leq \frac{1}{4}\|\phi-\psi\|_{E_{0}}+\frac{1}{8}\left[\|T \phi-T \psi\|_{E}+\|T \psi-\psi(c)\|_{E}\right] \\
& =\frac{1}{4}\|\phi-\psi\|_{E_{0}}+\frac{1}{8}\left[\frac{1}{16}\|\phi(c)-\psi(c)\|_{E}+\|T \psi-\psi(c)\|_{E}\right] \\
& \leq \frac{1}{4}\|\phi-\psi\|_{E_{0}}+\frac{1}{8}\left[\frac{1}{16}\|\phi-\psi\|_{E_{0}}+\|T \psi-\psi(c)\| \|_{E}\right] \\
& \leq \frac{1}{4} M(\phi, \psi)+\frac{1}{8}\left[\frac{1}{16} M(\phi, \psi)+M(\phi, \psi)\right] \\
& =\left[\frac{1}{4}+\frac{1}{128}+\frac{1}{8}\right] M(\phi, \psi) \\
& =\frac{49}{128} M(\phi, \psi)=\frac{49}{256} \eta(M(\phi, \psi)) .
\end{aligned}
$$

Therefore

$$
\eta\left(\|T \phi-T \psi\|_{E}\right)<\frac{49}{256} \eta(M(\phi, \psi)) .
$$

Case (ii): Suppose that $T \phi=\frac{\phi(c)}{8}$ and $T \psi=\frac{\psi(c)}{8}$.

$\overline{C l e a r l y}\|T \phi-T \psi\|_{E}=\frac{1}{8}\|\phi(c)-\psi(c)\|_{E}$ which implies that

$\eta\left(\|T \phi-T \psi\|_{E}\right)=\eta\left(\frac{1}{8}\|\phi(c)-\psi(c)\|_{E}\right)=\frac{1}{4}\|\phi(c)-\psi(c)\|_{E}$

$$
\begin{aligned}
& \leq \frac{1}{4}\left[\|\phi(c)-T \phi\|_{E}\right]+\frac{1}{4}\left[\|T \phi-\psi(c)\|_{E}\right] \\
& <\frac{1}{2}\|\phi-\psi\|_{E_{0}}+\frac{1}{4}\left[\|T \phi-\psi(c)\|_{E}\right](b y[63)
\end{aligned}
$$


Therefore

$$
\begin{aligned}
& \leq \frac{1}{2}\|\phi-\psi\|_{E_{0}}+\frac{1}{4}\left[\|T \phi-T \psi\|_{E}+\|T \psi-\psi(c)\|_{E}\right] \\
& =\frac{1}{2}\|\phi-\psi\|_{E_{0}}+\frac{1}{4}\left[\frac{1}{8}\|\phi(c)-\psi(c)\|\left\|_{E}+\right\| T \psi-\psi(c) \|_{E}\right] \\
& \leq \frac{1}{2}\|\phi-\psi\|_{E_{0}}+\frac{1}{4}\left[\frac{1}{8}\|\phi-\psi\|_{E_{0}}+\|T \psi-\psi(c)\|_{E}\right] \\
& \leq \frac{1}{2} M(\phi, \psi)+\frac{1}{4}\left[\frac{1}{8} M(\phi, \psi)+M(\phi, \psi)\right] \\
& =\left[\frac{1}{2}+\frac{1}{32}+\frac{1}{4}\right] M(\phi, \psi) \\
& =\frac{25}{32} M(\phi, \psi)=\frac{25}{64} \eta(M(\phi, \psi)) .
\end{aligned}
$$

$$
\eta\left(\|T \phi-T \psi\|_{E}\right)<\frac{25}{64} \eta(M(\phi, \psi)) .
$$

Case (iii): Suppose that $T \phi=\frac{\phi(c)}{16}$ and $T \psi=\frac{\psi(c)}{8}$.

$\overline{\text { Clearly } \| T} \phi-T \psi\left\|_{E}=\right\| \frac{\phi(c)}{16}-\frac{\psi(c)}{8} \|_{E}$ which implies that

$\eta\left(\|T \phi-T \psi\|_{E}\right)=\eta\left(\left\|\frac{\phi(c)}{16}-\frac{\psi(c)}{8}\right\|_{E}\right)=\left\|\frac{\phi(c)}{8}-\frac{\psi(c)}{4}\right\|_{E}$

$$
\begin{aligned}
& \leq\left\|\frac{\phi(c)}{8}-\frac{T \phi}{8}\right\|_{E}+\left\|\frac{T \phi}{8}-\frac{\psi(c)}{4}\right\|_{E} \\
& <\frac{1}{4}\|\phi-\psi\|_{E_{0}}+\left\|\frac{\phi(c)}{128}-\frac{\psi(c)}{128}\right\|_{E}+\left\|\frac{\psi(c)}{128}-\frac{\psi(c)}{4}\right\|_{E}(b y[63) \\
& \leq \frac{1}{4}\|\phi-\psi\|_{E_{0}}+\frac{1}{128}\|\phi-\psi\|_{E_{0}}+\frac{31}{128}\|\psi(c)\|_{E} \\
& <\frac{1}{4}\|\phi-\psi\|\left\|_{E_{0}}+\frac{1}{128}\right\| \phi-\psi\left\|_{E_{0}}+\frac{7}{8}\right\| \psi(c) \|_{E} \\
& =\frac{1}{4}\|\phi-\psi\|\left\|_{E_{0}}+\frac{1}{128}\right\| \phi-\psi\left\|_{E_{0}}+\right\| T \psi-\psi(c) \|_{E} \\
& \leq \frac{1}{4} M(\phi, \psi)+\frac{1}{128} M(\phi, \psi)+M(\phi, \psi) \\
& =\left[\frac{1}{4}+\frac{1}{128}+1\right] M(\phi, \psi) \\
& =\frac{161}{128} M(\phi, \psi)=\frac{161}{256} \eta(M(\phi, \psi)) .
\end{aligned}
$$

Therefore

$$
\eta\left(\|T \phi-T \psi\|_{E}\right)<\frac{161}{256} \eta(M(\phi, \psi)) .
$$

Case (iv): Suppose that $T \phi=\frac{\phi(c)}{8}$ and $T \psi=\frac{\psi(c)}{16}$.

$\overline{\text { Clearly } \| T} \phi-T \psi\left\|_{E}=\right\| \frac{\phi(c)}{8}-\frac{\psi(c)}{16} \|_{E}$ which implies that

$\eta\left(\|T \phi-T \psi\|_{E}\right)=\eta\left(\left\|\frac{\phi(c)}{8}-\frac{\psi(c)}{16}\right\|_{E}\right)=\left\|\frac{\phi(c)}{4}-\frac{\psi(c)}{8}\right\|_{E}$

$$
\begin{aligned}
& \leq\left\|\frac{\phi(c)}{4}-\frac{T \phi}{4}\right\|_{E}+\left\|\frac{T \phi}{4}-\frac{\psi(c)}{8}\right\|_{E} \\
& <\frac{1}{2}\|\phi-\psi\|_{E_{0}}+\left\|\frac{T \phi}{4}-\frac{\psi(c)}{8}\right\|_{E}(b y \underline{63)} \\
& \leq \frac{1}{2}\|\phi-\psi\|_{E_{0}}+\left\|\frac{\phi(c)}{32}-\frac{\psi(c)}{32}\right\|_{E}+\left\|\frac{\psi(c)}{32}-\frac{\psi(c)}{8}\right\|_{E} \\
& <\frac{1}{2}\|\phi-\psi\|_{E_{0}}+\frac{1}{32}\|\phi-\psi\|\left\|_{E_{0}}+\frac{3}{32}\right\| \psi(c) \|_{E} \\
& \leq \frac{1}{2}\|\phi-\psi\|_{E_{0}}+\frac{1}{32}\|\phi-\psi\|_{E_{0}}+\frac{15}{16}\|\psi(c)\|_{E} \\
& =\frac{1}{2}\|\phi-\psi\|_{E_{0}}+\frac{1}{32}\|\phi-\psi\|_{E_{0}}+\|T \psi-\psi(c)\|_{E} \\
& \leq \frac{1}{2} M(\phi, \psi)+\frac{1}{32} M(\phi, \psi)+M(\phi, \psi) \\
& =\left[\frac{1}{2}+\frac{1}{32}+1\right] M(\phi, \psi) \\
& =\frac{49}{32} M(\phi, \psi)=\frac{49}{64} \eta(M(\phi, \psi)) .
\end{aligned}
$$

Therefore

$$
\eta\left(\|T \phi-T \psi\|_{E}\right)<\frac{49}{64} \eta(M(\phi, \psi)) .
$$

We choose $\lambda=\max \left\{\frac{49}{256}, \frac{25}{64}, \frac{161}{256}, \frac{49}{64}\right\}$. Clearly $\lambda \in(0,1)$.

From (64), (65), (66) and (67) we get

$\eta\left(\|T \phi-T \psi\|_{E}\right)<\lambda \eta(M(\phi, \psi))$.

This implies that

$\lambda \eta(M(\phi, \psi))-\eta\left(\|T \phi-T \psi\|_{E}\right)>0$ and hence

$$
\zeta\left(\eta\left(\|T \phi-T \psi\|_{E}\right), \eta(M(\phi, \psi))\right)>0 .
$$

Therefore $T$ is a generalized Suzuki type $Z_{G, \eta}$-contraction with respect to $\zeta$. 
For any $n \in \mathbb{R}$, we define $\phi_{n}: I \rightarrow E$ by

$$
\phi_{n}(x)= \begin{cases}n x^{2} & \text { if } x \in\left[\frac{1}{2}, 1\right] \\ \frac{n}{x^{2}} & \text { if } x \in[1,2] .\end{cases}
$$

Clearly $\phi_{n} \in E_{0},\left\|\phi_{n}\right\|_{E_{0}}=\left\|\phi_{n}(c)\right\|_{E}$ and hence $\phi_{n} \in R_{c}$ for any $n \in \mathbb{R}$.

Let $\mathrm{F}_{0}=\left\{\phi_{n} \mid n \in \mathbb{R}\right\}$. Then $\mathrm{F}_{0} \subseteq R_{c}$ and $\mathrm{F}_{0}$ is algebraically closed with respect to the difference.

Therefore $T$ satisfies all the hypotheses of Corollary 4.2 and hence $\phi_{0} \in R_{c}$ is a PPF dependent fixed point of $T$.

\section{Acknowledgements}

The authors would like to thank the honorable referee for his/her valuable suggestions which helped us to improve the presentation of the paper.

\section{References}

[1] A.H. Ansari, Note on $\phi-\psi-$ contractive type mappings and related fixed point, The 2nd Regional Conference on Mathematics and Applications, Payame Noor University Tehran, (2014), 377-380.

[2] A.H. Ansari, J. Kaewcharoen, $C$ - class functions and fixed point theorems for generalized $\alpha-\eta-\psi-\phi-F-$ contraction type mappings in $\alpha-\eta$ complete metric spaces, J. Nonlinear Sci. Appl., 9 (6) (2016), 4177-4190.

[3] G.V.R. Babu, P.D. Sailaja , A fixed point theorem of Generalized Weakly contractive maps in Orbitally Complete Metric space, Thai J. Math., 9 (1) (2011), 1-10.

[4] G.V.R. Babu, G. Satyanarayana, M. Vinod Kumar, Properties of Razumikhin class of functions and PPF dependent fixed points of Weakly contractive type mappings, Bull. Int. Math. Virtual Institute, 9 (1) (2019), 65-72.

[5] G.V.R. Babu, M. Vinod Kumar, PPF dependent coupled fixed points via $C$-class functions, J. Fixed Point Theory, 2019 (2019), Article ID 7.

[6] B.C. Dhage, On some common fixed point theorems with PPF dependence in Banach spaces, J. Nonlinear Sci. Appl., 5 (2012), 220-232.

[7] S.R. Bernfeld, V. Lakshmikantham, Y.M. Reddy, Fixed point theorems of operators with PPF dependence in Banach spaces, Appl. Anal., 6 (4) (1977), 271-280.

[8] L. Cirić, S.M. Alsulami, P. Salimi, P. Vetro, PPF dependent fixed point results for triangular $\alpha_{c}-$ admissible mappings, Hindawi Publishing corporation, (2014), Article ID 673647, 10 pages.

[9] Z. Dirci, F.A. McRae, J. Vasundharadevi, Fixed point theorems in partially ordered metric spaces for operators with PPF dependence, Nonlinear Anal., 67 (2007), 641-647.

[10] A. Farajzadeh, A. Kaewcharoen, S. Plubtieng, PPF dependent fixed point theorems for multivalued mappings in Banach spaces, Bull. Iranian Math. Soc., 42 (6) (2016), 1583-1595.

[11] F. Khojasteh, Satish Shukla, S. Radenović, A new approach to the study of fixed point theory for simulation function, Filomat, 29 (6) (2015), 1189-1194.

[12] H. Quwagneh, M.S. MD Noorani, W. Shatanawi, H. Alsamir, Common fixed points for pairs of triangular $\alpha-$ admissible mappings, J. Nonlinear Sci. Appl., 10 (2017), 6192 - 6204.

[13] J. Harjani, B. Lopez, K. Sadarangani, Fixed point theorems for mixed monotone operators and applications to integral equations, Nonlinear Anal. 74 (2011), 1749-1760.

[14] N. Hussain, S. Khaleghizadeh, P. Salimi, F. Akbar, New Fixed Point Results with PPF dependence in Banach Spaces Endowed with a Graph, Abstr. Appl. Anal., (2013), Article ID 827205.

[15] E. Karapinar, Fixed points results via simulation functions, Filomat, 30 (8) (2016), 2343 - 2350.

[16] E. Karapinar, P. Kumam, P. Salimi, On a $\alpha-\psi$-Meir-Keeler contractive mappings, Fixed point theory Appl., (2013), Article Number 94 (2013).

[17] P. Kumum, D. Gopal, L. Budhia, A new fixed point theorem under Suzuki type $Z$-contraction mappings, J. Math. Anal., 8 (1) (2017), 113-119.

[18] M.A. Kutbi, W. Sintunavarat, On sufficient coniditons for the existence of Past-Present-Future dependent fixed point in Razumikhin class and application, Abstr. Appl Anal., (2014), Article ID 342684.

[19] X.L. Liu, A.H. Ansari, S. Chandok. S. Radenović, On some results in metric spaces using auxiliary simulation functions via new functions, J. Comput. Anal. Appl., 24 (6) (2018).

[20] M. Mursaleen, S.A. Mohiuddine, R.P. Agarwal, Coupled fixed point theorems for $\alpha-\psi$-contractive type mappings in partially ordered metric spaces, Fixed Point Theory Appl., (2012), Arcticle Number 228 (2012).

[21] A. Padcharoen, P. Kumum, P. Saipara, P. Chaipunya, Generalized Suzuki type $Z$-contraction in complete metric spaces, Kragujevac J. Math., 42 (3) (2018), 419-430.

[22] O. Popescu, Some new fixed point theorems for $\alpha$-Geraght contractive type maps in metric spaces, Fixed Point Theory Appl., (2014), Article Number 190 (2014). 
[23] S. Radenović, F. Vetro, J. Vujaković, An alternative and easy approach to fixed point results via simulation functions, Demonstr. Math., 50 (1) (2017), 223-230.

[24] A.C.M. Ran, M.C.B. Reurings, A fixed point theorem in partially ordered sets and some applications to matrix equations, Proc. Am. Math. Soc 132 (5) (2003), 1435-1443.

[25] A. R. Roldán-Lopez-de-Hierro, E. Karapınar, C. Roldán-Lopez-de-Hierro, J. Martines-Moreno, Coincidence point theorems on metric spaces via simulation functions, J. Comput. Appl. Math., 275 (2015), 345-355.

[26] B. Samet, C. Vetro, P. Vetro, Fixed point theorems for $\alpha-\psi$-contractive type mappings, Nonlinear Anal., 75 (4) (2012), $2154-2165$. 